\title{
The Weibull Generalized Exponential Distribution with Censored Sample: Estimation and Application on Real Data
}

\author{
Hisham M. Almongy $\mathbb{D}^{1},{ }^{1}$ Ehab M. Almetwally ${ }^{D},{ }^{2}$ Randa Alharbi $\left(\mathbb{D},{ }^{3}\right.$ Dalia Alnagar $\left(\mathbb{D},{ }^{3}\right.$ \\ E. H. Hafez $\mathbb{D}^{4}{ }^{4}$ and Marwa M. Mohie El-Din $\mathbb{D}^{5}$ \\ ${ }^{1}$ Applied Statistics and Insurance Department, Faculty of Commerce, Mansoura University, Mansoura, Egypt \\ ${ }^{2}$ Statistics Department, Faculty of Business Administration, Delta University of Science and Technology, Mansoura, Egypt \\ ${ }^{3}$ Statistics Department, University of Tabuk, Tabuk, Saudi Arabia \\ ${ }^{4}$ Mathematics Department, Faculty of Science, Helwan University, Cairo, Egypt \\ ${ }^{5}$ Mathematical and Natural Sciences Department, Faculty of Engineering, Egyptian Russian University, Badr, Egypt \\ Correspondence should be addressed to Ehab M. Almetwally; ehabxp_2009@hotmail.com
}

Received 3 January 2021; Revised 18 January 2021; Accepted 2 February 2021; Published 26 February 2021

Academic Editor: Ahmed Mostafa Khalil

Copyright ( 92021 Hisham M. Almongy et al. This is an open access article distributed under the Creative Commons Attribution License, which permits unrestricted use, distribution, and reproduction in any medium, provided the original work is properly cited.

\begin{abstract}
This paper is concerned with the estimation of the Weibull generalized exponential distribution (WGED) parameters based on the adaptive Type-II progressive (ATIIP) censored sample. Maximum likelihood estimation (MLE), maximum product spacing (MPS), and Bayesian estimation based on Markov chain Monte Carlo (MCMC) methods have been determined to find the best estimation method. The Monte Carlo simulation is used to compare the three methods of estimation based on the ATIIP-censored sample, and also, we made a bootstrap confidence interval estimation. We will analyze data related to the distribution about single carbon fiber and electrical data as real data cases to show how the schemes work in practice.
\end{abstract}

\section{Introduction}

The importance of any distribution comes from its flexibility, and one of the most important families is the Weibull family as it has many applications in industry, medicine, and many science fields. The first one that proposed the Weibull generalized family of distributions using the Weibull generator was done in [1]. The authors in [2] used the Weibull generalized family to generate the new distribution by assuming exponential distribution as a baseline distribution, which is denoted Weibull generalized exponential distribution (WGED). According to [2], the WGED is better than different distributions as generalized exponential distribution (see [3]), beta exponential distribution (see [4]), and the beta generalized exponential distribution (see [5]) in fitting many kinds of data. Almetwally et al. [6] discussed the estimation of the WGED parameters with progressive Type-II censoring (PTIIC) schemes by using the maximum likelihood and Bayesian estimation methods. The authors in [7] addressed the estimation of WGED parameters based on generalized order statistics, and they derived the submodels of generalized order statistics such as order statistics and record values. The authors in [8] introduced a heavy tailed exponential distribution by using the alpha power method for generalized continuous distribution. Teamah et al. [9] presented Fréchet-Weibull mixture exponential distribution along with a variety of statistical properties.

The most commonly used censoring schemes are Type-I censored (or time censored) and Type-II censored (or failure-censored). These two censoring schemes do not allow for units to be removed from the experiments while they are still alive. Progressive censoring is a more general censoring scheme that allows the units to be removed from the test (see [10]). Progressive censoring is useful in a life-testing experiment because it has the ability to remove life units from 
the experiment, so it saves time and money. Applications under progressive Type-II censoring (PTIIC) using different lifetime distributions have been discussed by many authors, for example, see [11-14]p and [15].

$\mathrm{Ng}$ et al. [16] suggested an adaptive Type-II progressive censoring scheme (ATIIPCS) in which the effective number of failures $m<n$ is fixed in advance and the progressive censoring scheme $R_{1}, \ldots, R_{m}$ is provided. Suppose that the experimenter fixes a time $T$, which represents the time of the experiment, but the test itself may be allowed to run overtime $T$. Let us denote that $m$ is completely observed failure times by $X_{i: m: n}, i=1, \ldots, m$. If the $\mathrm{m}^{\text {th }}$ progressive censored failure time occurs before time $T$, the experiment will be terminated at the time $X_{m: m: n}$. Otherwise, once the experiment time passes time $T$, but the number of observed failures has not reached $m$, we will terminate the experiment. Many authors had discussed applications under ATIIPCS using different lifetime distributions, for example, see [17-21] and [22].

$\mathrm{Ng}$ et al. [16] introduced PTIIC by using MLE. Almetwally et al. [20] introduced PTIIC by using MPS. Basu et al. [23] developed MPS estimator for a progressive hybrid Type-I censoring scheme with binomial removals. ElSherpieny et al. [15] introduced progressive Type-II hybrid censoring based on the MPS method with application for power Lomax distribution. Almetwally et al. [21] discussed the Weibull parameter estimation under PTIIC by using MPS and MLE methods. Maximum product spacing for the stress-strength model based on progressive Type-II hybridcensored samples with different cases has been obtained by [24]. Parameters of the extended odd Weibull exponential distribution are estimated under the progressive Type-II censoring scheme with random removal using the maximum product spacing and maximum likelihood estimation methods by [25]. The MCMC algorithm for the Bayesian estimation, it was introduced by [26]. For more information, see $[20,27-29]$ and [30].

Because of the importance of the Weibull distribution and ATIIPCS in reliability studies, we had considered the ATIIPCS applied to items whose lifetimes under design conditions are assumed to follow WGED under the ATIIPCS with the random removal. The removals from the test are considered by using the binomial distribution. MLE, MPS, and approximate confidence intervals (CI) of the estimates are presented. Bayesian estimates, percentile bootstrap CI, and bootstrap-t CI are obtained. Monte Carlo simulation study, as well as application to real data, is performed to illustrate the theoretical results.

The paper is organized as follows: Section 2 is devoted to model description and notations of the WGED parameters using the classical estimation method under APTIIC. In Section 3, we introduced the classical estimation method under APTIIC. In Section 4, we introduced the Bayesian estimation method under APTIIC. A simulation study is performed to illustrate the statistical properties of the parameters in Section 5. Two real data applications are analyzed in Section 6. Eventually, the concluded remarks are given in Section 7.

\section{Model Description and Notations}

Assume a random variable $X>0$ has WGED with a vector of parameter $\Theta=(\alpha, \gamma, \theta)$, and say that its cumulative distribution function (CDF) is given by

$$
F(x ; \Theta)=1-e^{-\alpha\left(e^{\gamma x}-1\right)^{\theta}} .
$$

The corresponding PDF is

$$
f(x ; \Theta)=\alpha \gamma \theta e^{\gamma x}\left(e^{\gamma x}-1\right)^{\theta-1} e^{-\alpha\left(e^{\gamma x}-1\right)^{\theta}} .
$$

The quantile function of the WGE distribution is

$$
x=\frac{1}{\gamma} \ln \left(1+\left[\frac{-1}{\alpha} \ln (1-u)\right]^{(1 / \theta)}\right), \quad 0<u<1 .
$$

In the APTIIC, the scheme can be described as follows.

Assume that we set $n$ independent observations placed on a life testing and the progressive censoring scheme $R_{i}, i=1,2, \ldots, m$. At the time of the first failure, $x_{1}, R_{1} \sim \operatorname{binomal}(n-m, p)$ units are randomly removed from the remaining $(n-1)$ surviving items. At the time of the second failure, $x_{2}, R_{2} \sim \operatorname{binomal}\left(n-m-R_{1}, p\right)$ units of the remaining $n-2-R_{1}$, units are randomly removed, and so on, the test continues until the $m^{\text {th }}$ failure at which time, and all the remaining $n-m-R_{1}-R_{2}-\cdots-R_{m-1}$ units are removed.

In APTIIC, the number of failures $m$, with removal probability $p$, and time $T$ are fixed given by the experimenter. Suppose that an individual unit was being removed from the test is independent of the others but with the same removal probability $p$. Then, the number of units removed at each failure time follows a binomial distribution which is, for $i=2,3, \ldots, m-1 \quad R_{i} \sim \operatorname{binomal}\left(n-m-\sum_{j=1}^{i-1} R_{j}, p\right)$ and $R_{m}=n-m-\sum_{j=1}^{m-1} R_{j}$. If the $m^{\text {th }}$ progressively censored failure time occurs before $T$, the experiment will be terminated at the time $X_{m: m: n}$. Otherwise, once the experimental time exceeds time $T$, but the number of observed failures has not reached $m$, we would terminate the experiment as soon as possible. The data form is as follows: $X_{1: m: n}<X_{2: m: n}<\ldots<X_{D: m: n}<T<\ldots<X_{m: m: n}$.

The number of units removed at each failure time assumed to follow a binomial distribution with the following probability mass function:

$$
\operatorname{Pr}\left(R_{1}=r_{1}\right)=\left(\begin{array}{c}
n-m \\
r_{1}
\end{array}\right) p^{r_{1}}(1-p)^{n-m-r_{1}} .
$$

While for, $i=2,3, \ldots, m-1$,

$$
\begin{aligned}
\operatorname{Pr} & \left(R_{i}=r_{i} \mid R_{i-1}=r_{i-1}, \ldots, R_{1}=r_{1}\right) \\
= & \left(\begin{array}{c}
n-m-\sum_{j=1}^{i-1} r_{j} \\
r_{i}
\end{array}\right) p^{r_{i}}(1-p)^{n-m-\sum_{j=1}^{i} r_{j}},
\end{aligned}
$$

where $0 \leq r_{i} \leq n-m-\sum_{j=1}^{i-1} r_{j}$. Furthermore, suppose that $R_{i}$ is independent of $X_{i: m: n}$ for all $i$. Then, the joint likelihood function can be found. 
That is,

$$
L\left(x_{i: m: n}, \Theta\right)=L_{1}\left(x_{i: m: n}, \Theta\right) \operatorname{Pr}(\mathbf{R}=\mathbf{r}),
$$

where $\operatorname{Pr}(\mathbf{R}=\mathbf{r})=\operatorname{Pr}\left(R_{1}=r_{1}, R_{2}=r_{2}, \ldots, R_{m-1}=r_{m-1}\right)$, i.e.,

$$
\operatorname{Pr}(\mathbf{R}=\mathbf{r})=\frac{(n-m) !}{\left(n-m-\sum_{j=1}^{m-1} r_{j}\right) ! \prod_{j=1}^{m-1} r_{j}} p^{\sum_{j=1}^{m-1} r_{j}}(1-p)^{(m-1)(n-m)-\sum_{j=1}^{m-1}(m-j) r_{j}}
$$

The MLE of $p$ can be derived by maximizing equation (6) directly. Hence, the MLE of $p$ is obtained by solving the following equation:

$$
\frac{\partial \ln L}{\partial p}=\frac{\sum_{i=1}^{m-1} r_{i}}{p}-\frac{(m-1)(n-m)-\sum_{i=1}^{m-1}(m-i) r_{i}}{1-p} .
$$

Hence,

$$
\widehat{p}=\frac{\sum_{i=1}^{m-1} r_{i}}{(m-1)(n-m)-\sum_{i=1}^{m-1}(m-i-1) r_{i}} .
$$

Since, $L_{1}\left(x_{i: m: n}, \Theta\right)$ does not involve the binomial parameter $p$, then the likelihood function under ATIIPCS can be written as

$$
L_{1}\left(x_{i: m: n}, \Theta\right)=A\left(\prod_{i=1}^{m} f\left(x_{i: m: n}, \Theta\right)\right)\left(\prod_{i=1}^{D}\left(1-F\left(x_{i: m: n}, \Theta\right)\right)^{R_{i}}\right) \cdot\left(1-F\left(x_{m: m: n}, \Theta\right)\right)^{n-m-\sum_{i=1}^{D} R_{i}}
$$

The joint MPS under ATIIPCS can be written in the

$$
S\left(x_{i: m: n}, \Theta\right)=S_{1}\left(x_{i: m: n}, \Theta\right) \operatorname{Pr}(\mathbf{R}=\mathbf{r}) \text {, where }
$$
same manner as

$$
S_{1}\left(x_{i: m: n}, \Theta\right)=A \prod_{i=1}^{m+1}\left(D_{i: m: n}, \Theta\right)\left(\prod_{i=1}^{D}\left(1-F\left(x_{i: m: n}, \Theta\right)\right)^{R_{i}}\right) \cdot\left(1-F\left(x_{m: m: n}, \Theta\right)\right)^{n-m-\sum_{i=1}^{D} R_{i}} \text {, }
$$

where $\mathrm{A}$ is a constant that does not depend on parameters and

$$
D_{i: m: n}=\left\{\begin{array}{l}
F\left(x_{1: m: n}\right) \\
F\left(x_{i: m: n}\right)-F\left(x_{(i-1): m: n}\right), \quad i=2, \ldots m . \\
1-F\left(x_{m: m: n}\right)
\end{array}\right.
$$

\section{The Classical Estimation Method under ATIIPCS}

This section deals with MLE and MPS methods of the parameters WGED based on the ATIIPCS data with binomial removal.

3.1. MLE Method. Using equation (10), the likelihood function for WGED based on ATIIPCS can be written as

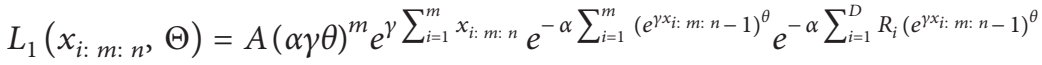

$$
\begin{aligned}
& \times e^{-\alpha\left(n-m-\sum_{i=1}^{D} R_{i}\right)\left(e^{\gamma x_{m: m: n}}-1\right)^{\theta}} \prod_{i=1}^{m}\left(e^{\gamma x_{i: m: n}}-1\right)^{\theta-1},
\end{aligned}
$$

where $A=n\left(n-R_{1}-1\right) \cdots\left(n-\sum_{i=1}^{m-1} R_{i}-(m-1)\right)$ is a constant which does not depend on the parameters.
The natural logarithm of the likelihood function equation can be obtained as follows: 


$$
\begin{aligned}
\ln L_{1}\left(x_{i: m: n}, \Theta\right)= & \ln A+m \ln (\alpha \gamma \theta)+\gamma \sum_{i=1}^{m} x_{i: m: n}+(\theta-1) \sum_{i=1}^{m} \ln \left(e^{\gamma x_{i: m: n}}-1\right)-\alpha \sum_{i=1}^{m}\left(e^{\gamma x_{i: m: n}}-1\right)^{\theta} \\
& -\alpha \sum_{i=1}^{D} R_{i}\left(e^{\gamma x_{i: m: n}}-1\right)^{\theta}-\alpha\left(n-m-\sum_{i=1}^{D} R_{i}\right)\left(e^{\gamma x_{m: m: n}}-1\right)^{\theta}
\end{aligned}
$$

For convenience, let $l(\Theta)=\ln L_{1}\left(x_{i: m: n}, \Theta\right)$; hence, the partial derivatives of equation (14) are given as follows:

$$
\begin{aligned}
\frac{\partial l(\Theta)}{\partial \alpha}= & \frac{m}{\alpha}-\sum_{i=1}^{m}\left(e^{\gamma x_{i: m: n}}-1\right)^{\theta}-\sum_{i=1}^{D} R_{i}\left(e^{\gamma x_{i: m: n}}-1\right)^{\theta}-\left(n-m-\sum_{i=1}^{D} R_{i}\right)\left(e^{\gamma x_{m: m: n}}-1\right)^{\theta}, \\
\frac{\partial l(\Theta)}{\partial \gamma}= & \frac{m}{\gamma}+\sum_{i=1}^{m} x_{i: m: n}-\alpha \theta\left(n-m-\sum_{i=1}^{D} R_{i}\right) x_{m: m: n} e^{\gamma x_{m: m: n}}\left(e^{\gamma x_{m: m: n}}-1\right)^{\theta-1} \\
& -\theta \alpha \sum_{i=1}^{m} x_{i: m: n} e^{\gamma x_{i: m: n}}\left(e^{\gamma x_{i: m: n}}-1\right)^{\theta-1}+(\theta-1) \sum_{i=1}^{m} \frac{x_{i: m: n} e^{\gamma x_{i: m: n}}}{\left(e^{\gamma x_{i: m: n}}-1\right)}-\theta \alpha \sum_{i=1}^{D} R_{i} x_{i: m: n} e^{\gamma x_{i: m: n}}\left(e^{\gamma x_{i}: m: n}-1\right)^{\theta-1},
\end{aligned}
$$

and

$$
\begin{aligned}
\frac{\partial l(\Theta)}{\partial \theta}= & \frac{m}{\theta}+\sum_{i=1}^{m} \ln \left(e^{\gamma x_{i: m: n}}-1\right)-\alpha\left(n-m-\sum_{i=1}^{D} R_{i}\right)\left(e^{\gamma x_{m: m: n}}-1\right)^{\theta} \ln \left(e^{\gamma x_{m: m: n}}-1\right) \\
& -\alpha \sum_{i=1}^{m}\left(e^{\gamma x_{i: m: n}}-1\right)^{\theta} \ln \left(e^{\gamma x_{i: m: n}}-1\right)-\alpha \sum_{i=1}^{D} R_{i}\left(e^{\gamma x_{i: m: n}}-1\right)^{\theta} \ln \left(e^{\gamma x_{i: m: n}}-1\right) .
\end{aligned}
$$

The MLE of $\Theta$ for the WGED parameters is the solution of equations (15), (16), and (17) by using the Newton-Raphson method. Furthermore, the asymptotic CI (ACI) can be approximated numerically by inverting Fisher's information matrix. Thus, the 95\% ACI for $\alpha, \gamma$, and $\theta$ is easily obtained, respectively, as $\widehat{\alpha} \pm$ $Z_{0.025} \sqrt{\operatorname{Var}(\widehat{\alpha})}, \hat{\gamma} \pm Z_{0.025} \sqrt{\operatorname{Var}(\widehat{\gamma})}$ and $\widehat{\theta} \pm Z_{0.025} \sqrt{\operatorname{Var}(\widehat{\theta})}$.

3.2. MPS Method. With the use of equation (11), the product spacing function for WGED based on ATIIPCS can be written as

$$
\begin{aligned}
S_{1}\left(x_{i: m: n}, \Theta\right)= & A\left(1-e^{-\alpha\left(e^{\gamma x_{1: m: n}}-1\right)^{\theta}}\right) e^{-\alpha\left(1+\left(n-m-\sum_{i=1}^{D} R_{i}\right)\right)\left(e^{\gamma x_{m: m: n}}-1\right)^{\theta}} \\
& \left.\times e^{-\alpha \sum_{i=1}^{D} R_{i}\left(e^{\gamma x_{i: m: n-1}}\right)^{\theta}} \prod_{i=2}^{m}\left(e^{-\alpha\left(e^{\gamma x}(i-1): m: n-1\right.}\right)^{\theta}-e^{-\alpha\left(e^{\gamma x_{i: m: n}}-1\right)^{\theta}}\right),
\end{aligned}
$$

where $A$ is a constant which does not depend on the parameters. The natural logarithm of the product spacing function is 


$$
\begin{aligned}
\ln S_{1}\left(x_{i: m: n}, \Theta\right)= & \ln A+\ln \left(1-e^{-\alpha\left(e^{\gamma x_{1}: m: n}-1\right)^{\theta}}\right)-\alpha \sum_{i=1}^{D} R_{i}\left(e^{\gamma x_{i: m: n}}-1\right)^{\theta} \\
& \left.+\sum_{i=2}^{m} \ln \left(e^{-\alpha\left(e^{\gamma x}(i-1): m: n-1\right.}\right)^{\theta}-e^{-\alpha\left(e^{\gamma x_{i}: m: n-1}\right)^{\theta}}\right)-\alpha\left(1+\left(n-m-\sum_{i=1}^{D} R_{i}\right)\right)\left(e^{\gamma x_{m: m: n}}-1\right)^{\theta} .
\end{aligned}
$$

Let $s(\Theta)=\ln S_{1}\left(x_{i: m: n}, \Theta\right)$, then the partial derivatives by the MPS method of equation (19) are given as follows:

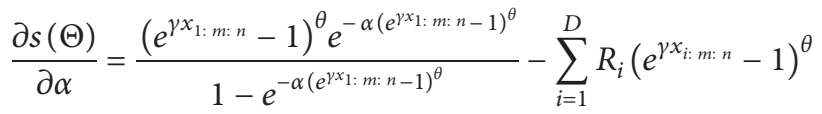

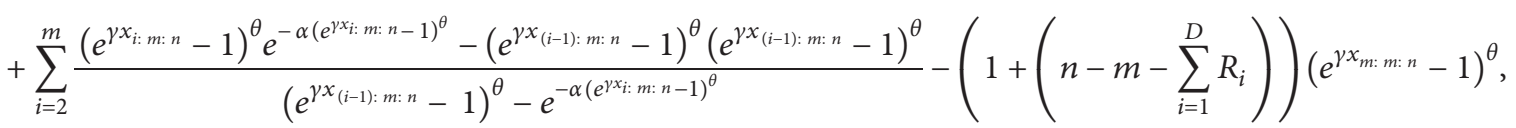

$$
\begin{aligned}
& \frac{\partial s(\Theta)}{\partial \gamma}=\frac{\alpha \theta x_{1: m: n} e^{\gamma x_{1: m: n}}\left(e^{\gamma x_{1: m: n}}-1\right)^{\theta-1} e^{-\alpha\left(e^{\left.\gamma x_{1}: m: n-1\right)^{\theta}}\right.}}{1-e^{-\alpha\left(e^{\left.\gamma x_{1}: m: n-1\right)^{\theta}}\right.}} \\
& -\alpha \theta \sum_{i=1}^{D} x_{i: m: n} e^{\gamma x_{i: m: n}} R_{i}\left(e^{\gamma x_{i: m: n}}-1\right)^{\theta-1}+\alpha \theta \sum_{i=2}^{m} \frac{x_{i: m: n} e^{\gamma x_{i: m: n}}\left(e^{\gamma x_{i: m: n}}-1\right)^{\theta-1} e^{-\alpha\left(e^{\gamma x_{i}: m: n}-1\right)^{\theta}}}{\left.e^{-\alpha\left(e^{p x}(i-1): m: n\right.}-1\right)^{\theta}}-e^{-\alpha\left(e^{\left.\gamma x_{i}: m: n-1\right)^{\theta}}\right.} \\
& -\alpha \theta \sum_{i=2}^{m} \frac{x_{(i-1): m: n} e^{\gamma x_{(i-1): m: n}}\left(e^{\gamma x_{(i-1): m: n}}-1\right)^{\theta-1} e^{-\alpha\left(e^{\gamma x(i-1): m: n-1}\right)^{\theta}}}{e^{-\alpha\left(e^{\gamma x}(i-1): m: n-1\right)^{\theta}}-e^{-\alpha\left(e^{\left.p x_{i: m}: n-1\right)^{\theta}}\right.}} \\
& -\alpha \theta\left(1+\left(n-m-\sum_{i=1}^{D} R_{i}\right)\right) x_{m: m: n} e^{\gamma x_{m: m: n}}\left(e^{\gamma x_{m: m: n}}-1\right)^{\theta-1},
\end{aligned}
$$

and

$$
\begin{aligned}
& \frac{\partial l(\Theta)}{\partial \theta}=\frac{\alpha \ln \left(e^{\gamma x_{1: m: n}}-1\right)\left(e^{\gamma x_{1: m: n}}-1\right)^{\theta} e^{-\alpha\left(e^{\gamma x_{1}: m: n}-1\right)^{\theta}}}{1-e^{-\alpha\left(e^{\gamma x_{1}: m: n}-1\right)^{\theta}}}-\alpha \sum_{i=1}^{D} R_{i}\left(e^{\gamma x_{i: m: n}}-1\right)^{\theta} \ln \left(e^{\gamma x_{i: m: n}}-1\right) \\
& +\sum_{i=2}^{m} \frac{\alpha\left(e^{\gamma x_{i: m: n}}-1\right)^{\theta} \ln \left(e^{\gamma x_{i: m: n}}-1\right) e^{-\alpha\left(e^{\left.\gamma x_{i: m}: n-1\right)^{\theta}}\right.}}{e^{-\alpha\left(e^{\gamma x}(i-1): m: n-1\right)^{\theta}}-e^{-\alpha\left(e^{\left.\gamma x_{i}: m: n-1\right)^{\theta}}\right.}}-\sum_{i=2}^{m} \frac{\alpha\left(e^{\gamma x_{(i-1): m: n}}-1\right)^{\theta} \ln \left(e^{\gamma x_{(i-1): m: n}}-1\right) e^{-\alpha\left(e^{\gamma x(i-1): m: n}-1\right)^{\theta}}}{e^{-\alpha\left(e^{\gamma x}(i-1): m: n-1\right)^{\theta}}-e^{-\alpha\left(e^{\left.\gamma x_{i}: m: n-1\right)^{\theta}}\right.}} \\
& -\alpha\left(1+\left(n-m-\sum_{i=1}^{D} R_{i}\right)\right) \ln \left(e^{\gamma x_{m: m: n}}-1\right)\left(e^{\gamma x_{m: m: n}}-1\right)^{\theta} .
\end{aligned}
$$

The MPS estimates for the WGED parameters can be obtained using the Newton-Raphson method. Moreover, we use the approximate $95 \% \mathrm{CI}$ for $\alpha, \gamma$, and $\theta$, respectively, as follows: $\widehat{\alpha} \pm Z_{0.025} \sqrt{\operatorname{Var}(\widehat{\alpha})}, \hat{\gamma} \pm Z_{0.025} \sqrt{\operatorname{Var}(\widehat{\gamma})}$ and $\hat{\theta} \pm Z_{0.025}$ $\sqrt{\operatorname{Var}(\hat{\theta})}$.
Also, we propose different bootstrap CIs of population parameters under the MLE method based on ATIIPCS data with binomial removal for the WGED as a bootstrap percentile (BP) and bootstrap-t (BT). For more information about this algorithm, see [31, 32] and [15]. 


\section{Bayesian Estimation}

In this section, we consider the Bayesian estimation for the parameters of WGED based on ATIIPCS under the assumption that the random variables $\Theta=(\alpha, \gamma, \theta)$ have an independent gamma prior distribution. Assume that $\alpha \sim \operatorname{Gamma}\left(a_{1}, b_{1}\right), \quad \quad \gamma \sim \operatorname{Gamma}\left(a_{2}, b_{2}\right), \quad$ and $\theta \sim \operatorname{Gamma}\left(a_{3}, b_{3}\right)$ (see $[6,33]$ ); the prior joint density of $\alpha . \gamma$, and $\theta$ can be written as

$$
\pi(\Theta) \propto \alpha^{a_{1}-1} e^{-\alpha b_{1}} \gamma^{a_{2}-1} e^{-\gamma b_{2}} \theta^{a_{3}-1} e^{-\theta b_{3}} .
$$

The posterior likelihood can be represented to be proportional to the product of the likelihood given in equation (13) and the joint prior's densities given by equation (22). That is,

$$
\Pi\left(\Theta \mid x_{i: m: n}\right) \propto L\left(x_{i: m: n} \mid \Theta\right) \pi(\Theta) .
$$

Then, the posterior joint density of $\Theta$ is

$$
\begin{aligned}
\Pi\left(\Theta \mid x_{i: m: n}\right) \propto & \alpha^{m+a_{1}-1} \gamma^{m+a_{2}-1} \theta^{m+a_{3}-1} e^{\gamma\left(\sum_{i=1}^{m} x_{i: m: n}-b_{2}\right)} \\
& \cdot \prod_{i=1}^{m}\left(e^{\gamma x_{i: m: n}}-1\right)^{\theta-1} \\
& \times e^{-\alpha\left(n-m-\sum_{i=1}^{D} R_{i}\right)\left(e^{\left.\gamma x_{m: m: n}-1\right)^{\theta}}\right.} \\
& \cdot e^{-\alpha\left(\sum _ { i = 1 } ^ { m } \left(e^{\left.\gamma x_{i: m: n}-1\right)^{\theta}+\sum_{i=1}^{D} R_{i}\left(e^{\left.\gamma x_{i: m: n}-1\right)^{\theta}+b_{1}}\right)} e^{-\theta b_{3}}\right.\right.}
\end{aligned}
$$

Using the squared error loss function (SE), the Bayesian estimators of the parameters $\Theta$ are obtained as follows:

$$
\begin{aligned}
& \tilde{\alpha}=\int_{0}^{\infty} \alpha \Pi\left(\alpha \mid \theta, \gamma, x_{i: m: n}\right) \mathrm{d} \alpha, \\
& \tilde{\gamma}=\int_{0}^{\infty} \gamma \Pi\left(\gamma \mid \theta, \alpha, x_{i: m: n}\right) \mathrm{d} \gamma, \\
& \tilde{\theta}=\int_{0}^{\infty} \theta \Pi\left(\theta \mid \gamma, \alpha, x_{i: m: n}\right) \mathrm{d} \theta .
\end{aligned}
$$

These integrals are very hard to be solved analytically, so that the MCMC approach will be used. An important subclass of the MCMC techniques is Gibbs sampling and more general Metropolis-within-Gibbs samplers. Metropolis et al. [26] were the first to introduce this algorithm.

The Metropolis-Hastings ( $\mathrm{MH}$ ) algorithm and the Gibbs sampling are the two most popular examples of an MCMC method. It is similar to acceptance-rejection sampling; the $\mathrm{MH}$ algorithm considers that, to each iteration of the algorithm, a candidate value can be generated from a proposal distribution. The $\mathrm{MH}$ algorithm generates a sequence of draws from WGED under ATIIPCS as follows: Algorithm 1 and 2 .

According to [34], we obtain Bayes credible intervals of the parameters $\Theta=(\alpha, \gamma, \theta)$ as follows:

Furthermore, for different bootstrap CIs of population parameters under the Bayesian estimation method based on
ATIIPCS data with binomial removal for the WGED as BP and BT, see Tables 1 and 2 .

\section{Simulation Study}

In this section, Monte Carlo simulation was done for comparison between maximum likelihood and Bayesian estimation methods under censoring scheme, for estimating parameters of WGED in a lifetime by $R$ language. Monte Carlo experiments were carried out based on the following data generated from WGED, where $X$ is distributed as WGED for different shape parameters:

Case -1. True values for $(\alpha=3.5, \gamma=3, \theta=2.5$, and $T=0.25)$.

Case-2. True values for $(\alpha=0.5, \gamma=2, \theta=2.5$, and $T=0.5)$.

We made this simulation using different sample sizes $n=50$ and 150 , different censored sample sizes $m$, and set of different sample schemes, and $p$ is $0.25,0.5$, and 0.75 .

We could define the best scheme as the scheme, which minimizes the mean squared error $(\operatorname{MSE}(\Theta))$, bias of estimation, and length of CI (L.CI) of the estimator. For Bayes confidence credible intervals, denoted as CCI, the CI of MLE, MPS, and Bayesian estimation and associated CI are calculated.

Relative efficiency is calculated as follows: $\mathrm{RE}_{1}=(\mathrm{MS}$ (MLE)/MSE (MPS)), $\mathrm{RE}_{2}=(\mathrm{MSE}(\mathrm{MLE}) / \mathrm{MSE}$ (Bayesian)) and $\mathrm{RE}_{3}=(\mathrm{MSE}(\mathrm{MPS}) / \mathrm{MSE}$ (Bayesian) $)$.

We conclude remarks on the simulation as follows:

(1) The simulation outcomes are recorded in Tables 1-4. The following concluding remakes are noticed based on these tables as follows.

(2) As $m$ increases with fixed values of $n$ and $p$, the Bias, MSE, and the L.CI associated with the parameter estimates decrease for both methods of estimation.

(3) For fixed values of $m$ and $p$, the Bias, MSE, and the L.CI associated with the parameter estimates decrease for both methods of estimation as $n$ increases.

(4) For fixed $n, \mathrm{~m}$, and as $p$ increases until 0.5, the Bias, MSE, and the L.CI associated with the parameter estimates decrease for the MLE and Bayesian methods.

(5) In approximately most situations, we notice that the measures of Bayesian estimates are more accurate than the measures of MLE and MPS estimates.

\section{Application of Real Data}

In this section, we will apply the numerical results of the parameter estimation of WGED under ATIIPCS on two cases of real data, namely, electric data and carbon fibers data.

6.1. Electric Data. Balakrishnan and Cramer [35] discussed this electric data, which are 19 failure times (in minutes) for an insulating fluid between two electrodes subject to a voltage of $34 \mathrm{KV}: 0.19,0.78,0.96,1.31,2.78,3.16,4.15,4.67$, $4.85,6.50,7.35,8.01,8.27,12.06,31.75,32.52,33.91,36.71$, and 72.89. We computed the Kolmogorov-Smirnov (KS) distance $(D)$ between the fitted and the empirical 
TABLE 1: The length of the different intervals of the WGED parameters based on ATIIPCS with various random removal of removing items, in case- 1 .

\begin{tabular}{|c|c|c|c|c|c|c|c|c|c|c|c|}
\hline \multicolumn{12}{|c|}{$\alpha=3.5, \gamma=3, \theta=2.5$, and $T=0.25$} \\
\hline \multirow{2}{*}{$P$} & \multirow{2}{*}{$(n, m)$} & & \multicolumn{3}{|c|}{ MLE } & \multicolumn{3}{|c|}{ MPS } & \multicolumn{3}{|c|}{ Bayesian } \\
\hline & & & $\mathrm{ACI}$ & BT & $\mathrm{BP}$ & ACI & BT & $\mathrm{BP}$ & CCI & BT & $\mathrm{BP}$ \\
\hline \multirow{6}{*}{0.25} & \multirow{3}{*}{$(50,30)$} & $\alpha$ & 5.0281 & 0.1518 & 0.2139 & 2.8130 & 0.0920 & 0.0876 & 2.3053 & 0.0932 & 0.0859 \\
\hline & & $\gamma$ & 1.8528 & 0.0591 & 0.0653 & 1.4089 & 0.0435 & 0.0463 & 0.9311 & 0.0461 & 0.0451 \\
\hline & & $\theta$ & 1.6220 & 0.0536 & 0.0514 & 1.4535 & 0.0465 & 0.0460 & 1.5228 & 0.0462 & 0.0472 \\
\hline & \multirow{3}{*}{$(50,45)$} & $\alpha$ & 2.2339 & 0.0685 & 0.0695 & 2.7294 & 0.0838 & 0.0880 & 1.7615 & 0.0889 & 0.0865 \\
\hline & & $\gamma$ & 1.1134 & 0.0353 & 0.0371 & 1.3269 & 0.0433 & 0.0416 & 0.7910 & 0.0416 & 0.0430 \\
\hline & & $\theta$ & 1.1839 & 0.0377 & 0.0379 & 1.1152 & 0.0366 & 0.0362 & 1.1347 & 0.0360 & 0.0365 \\
\hline \multirow{6}{*}{0.5} & \multirow{3}{*}{$(50,30)$} & $\alpha$ & 2.6530 & 0.0840 & 0.0787 & 2.8534 & 0.0894 & 0.0958 & 2.0821 & 0.0860 & 0.0950 \\
\hline & & $\gamma$ & 1.1874 & 0.0383 & 0.0366 & 1.3609 & 0.0442 & 0.0454 & 0.9093 & 0.0430 & 0.0420 \\
\hline & & $\theta$ & 1.6475 & 0.0527 & 0.0535 & 1.5203 & 0.0493 & 0.0464 & 1.5795 & 0.0483 & 0.0471 \\
\hline & \multirow{3}{*}{$(50,45)$} & $\alpha$ & 5.4329 & 0.1721 & 0.2769 & 2.9217 & 0.0933 & 0.0938 & 1.8254 & 0.0985 & 0.0877 \\
\hline & & $\gamma$ & 1.9696 & 0.0622 & 0.0708 & 1.4695 & 0.0456 & 0.0483 & 0.7633 & 0.0484 & 0.0488 \\
\hline & & $\theta$ & 1.2258 & 0.0388 & 0.0379 & 1.1214 & 0.0353 & 0.0359 & 1.1382 & 0.0373 & 0.0331 \\
\hline \multirow{6}{*}{0.75} & \multirow{3}{*}{$(50,30)$} & $\alpha$ & 3.8944 & 0.1229 & 0.1380 & 2.7236 & 0.0825 & 0.0884 & 2.2240 & 0.0887 & 0.0876 \\
\hline & & $\gamma$ & 1.5368 & 0.0479 & 0.0502 & 1.2724 & 0.0398 & 0.0403 & 0.9838 & 0.0415 & 0.0396 \\
\hline & & $\theta$ & 1.6526 & 0.0534 & 0.0523 & 1.4974 & 0.0462 & 0.0474 & 1.5582 & 0.0484 & 0.0454 \\
\hline & \multirow{3}{*}{$(50,45)$} & $\alpha$ & 2.3122 & 0.0711 & 0.0703 & 2.6791 & 0.0879 & 0.0871 & 1.7218 & 0.0855 & 0.0846 \\
\hline & & $\gamma$ & 1.0808 & 0.0343 & 0.0336 & 1.2658 & 0.0422 & 0.0409 & 0.7405 & 0.0410 & 0.0421 \\
\hline & & $\theta$ & 1.1806 & 0.0351 & 0.0365 & 1.1096 & 0.0352 & 0.0354 & 1.1346 & 0.0343 & 0.0364 \\
\hline \multirow{6}{*}{0.25} & \multirow{3}{*}{$(150,90)$} & $\alpha$ & 4.5602 & 0.2127 & 0.2406 & 3.9598 & 0.1329 & 0.1204 & 2.4745 & 0.1243 & 0.1230 \\
\hline & & $\gamma$ & 1.9321 & 0.0568 & 0.0732 & 1.0677 & 0.0339 & 0.0363 & 0.6765 & 0.0356 & 0.0345 \\
\hline & & $\theta$ & 0.8876 & 0.0278 & 0.0279 & 0.7957 & 0.0244 & 0.0270 & 0.8081 & 0.0244 & 0.0244 \\
\hline & \multirow{3}{*}{$(150,130)$} & $\alpha$ & 1.0331 & 0.0341 & 0.0341 & 1.3191 & 0.0425 & 0.0429 & 1.6192 & 0.0425 & 0.0405 \\
\hline & & $\gamma$ & 0.4880 & 0.0157 & 0.0158 & 0.6561 & 0.0213 & 0.0203 & 0.5414 & 0.0212 & 0.0216 \\
\hline & & $\theta$ & 0.6758 & 0.0206 & 0.0216 & 0.6632 & 0.0224 & 0.0213 & 0.6589 & 0.0209 & 0.0217 \\
\hline \multirow{6}{*}{0.5} & \multirow{3}{*}{$(150,90)$} & $\alpha$ & 3.8998 & 0.1207 & 0.1221 & 4.0729 & 0.1314 & 0.1266 & 2.1824 & 0.1237 & 0.1322 \\
\hline & & $\gamma$ & 1.0967 & 0.0353 & 0.0349 & 1.1471 & 0.0356 & 0.0351 & 0.6868 & 0.0368 & 0.0377 \\
\hline & & $\theta$ & 0.8480 & 0.0272 & 0.0257 & 0.8184 & 0.0261 & 0.0254 & 0.8331 & 0.0257 & 0.0269 \\
\hline & \multirow{3}{*}{$(150,130)$} & $\alpha$ & 3.1909 & 0.1261 & 0.1141 & 1.2513 & 0.0414 & 0.0412 & 1.7332 & 0.0383 & 0.0400 \\
\hline & & $\gamma$ & 2.5951 & 0.0708 & 0.1221 & 0.6555 & 0.0212 & 0.0212 & 0.5664 & 0.0217 & 0.0210 \\
\hline & & $\theta$ & 0.7472 & 0.0253 & 0.0240 & 0.6617 & 0.0214 & 0.0205 & 0.6627 & 0.0216 & 0.0213 \\
\hline \multirow{6}{*}{0.75} & \multirow{3}{*}{$(150,90)$} & $\alpha$ & 4.7341 & 0.1543 & 0.1553 & 3.9317 & 0.1307 & 0.1306 & 2.3038 & 0.1210 & 0.1259 \\
\hline & & $\gamma$ & 2.0889 & 0.0688 & 0.0726 & 1.0134 & 0.0324 & 0.0330 & 0.9657 & 0.0334 & 0.0331 \\
\hline & & $\theta$ & 0.9048 & 0.0293 & 0.0305 & 0.8221 & 0.0269 & 0.0266 & 0.8598 & 0.0255 & 0.0269 \\
\hline & \multirow{3}{*}{$(150,130)$} & $\alpha$ & 1.2868 & 0.0416 & 0.0388 & 1.6027 & 0.0485 & 0.0517 & 1.6520 & 0.0486 & 0.0521 \\
\hline & & $\gamma$ & 0.6521 & 0.0208 & 0.0209 & 0.8475 & 0.0269 & 0.0272 & 0.5677 & 0.0270 & 0.0284 \\
\hline & & $\theta$ & 0.6765 & 0.0201 & 0.0213 & 0.6669 & 0.0212 & 0.0213 & 0.6599 & 0.0215 & 0.0211 \\
\hline
\end{tabular}

distribution functions for the data to be 0.16538 , and the corresponding $p$ value is 0.6182 . Figure 1 discusses the plot of the max distance between the two CDF curves, histogram, PP-plot, and QQ-plot for WGED. Therefore, it indicates that WGED can be fitted to the electric data set. Table 5 shows the estimation of parameters and standard error (St.E) for complete electric data. Table 6 displays the sample of progressive Type-II censored data with $R$ removal for electric data. Table 7 gives the estimation of parameters and standard error under the censored sample for electric data.

Histogram plot, approximate marginal posterior density, and MCMC convergence of $\alpha, \gamma$, and $\theta$ are represented in Figure 2.

Histogram plot, approximate marginal posterior density, and MCMC convergence of $\alpha, \gamma$, and $\theta$ are represented in Figure 3.
6.2. Carbon Fiber Data. Bader and Priest [36] discussed carbon fibers of 69 observed failure times. These data sets represent the strength of items measured in GPA for single carbon fibers and impregnated 1000 carbon fiber tows. We computed the KS test distance is 0.07408 , and the corresponding $p$ value is 0.8605 . Therefore, it indicates that WGED can provide a good fit for the data set by using empirical cumulative distribution function (ECDF), histogram, PP-plot, and QQ-plot for carbon fiber data in Figure 4. Table 8 shows estimation of parameters and standard error for complete carbon fiber data.

Histogram plot, approximate marginal posterior density, and MCMC convergence of $\alpha, \gamma$, and $\theta$ are represented for carbon fiber data in Figure 5.

The data are under progressive censoring with random removal when $p=0.25$ and $m=20$. Then, the $R$ is $10,7,9,7,0$, 
TABLE 2: The length of the different intervals of the WGED parameters based on ATIIPCS with various random removal of removing items, in case-2.

\begin{tabular}{|c|c|c|c|c|c|c|c|c|c|c|c|}
\hline \multicolumn{12}{|c|}{$\alpha=0.5, \gamma=2, \theta=2.5$, and $T=0.5$} \\
\hline \multirow{2}{*}{$P$} & \multirow{2}{*}{$(n, m)$} & & \multicolumn{3}{|c|}{ MLE } & \multicolumn{3}{|c|}{ MPS } & \multicolumn{3}{|c|}{ Bayesian } \\
\hline & & & ACI & BT & $\mathrm{BP}$ & ACI & BT & $\mathrm{BP}$ & CCI & BT & $\mathrm{BP}$ \\
\hline \multirow{6}{*}{0.25} & \multirow{3}{*}{$(50,30)$} & $\alpha$ & 2.8215 & 0.1226 & 0.1379 & 1.5409 & 0.0698 & 0.0678 & 1.5324 & 0.0663 & 0.0696 \\
\hline & & $\gamma$ & 2.6097 & 0.1112 & 0.1318 & 1.3167 & 0.0602 & 0.0602 & 1.5651 & 0.0588 & 0.0588 \\
\hline & & $\theta$ & 1.6916 & 0.0711 & 0.0735 & 1.5437 & 0.0631 & 0.0712 & 1.5893 & 0.0705 & 0.0680 \\
\hline & \multirow{3}{*}{$(50,45)$} & $\alpha$ & 1.4167 & 0.1159 & 0.1250 & 1.7756 & 0.1457 & 0.1508 & 1.3004 & 0.1481 & 0.1512 \\
\hline & & $\gamma$ & 0.9602 & 0.0860 & 0.0785 & 1.0800 & 0.0906 & 0.0877 & 0.9251 & 0.0945 & 0.0976 \\
\hline & & $\theta$ & 1.0417 & 0.0892 & 0.0843 & 1.0036 & 0.0800 & 0.0840 & 1.0116 & 0.0835 & 0.0840 \\
\hline \multirow{6}{*}{0.5} & \multirow{3}{*}{$(50,30)$} & $\alpha$ & 1.6983 & 0.0896 & 0.0875 & 1.7943 & 0.0881 & 0.0837 & 1.2839 & 0.0921 & 0.0836 \\
\hline & & $\gamma$ & 1.1643 & 0.0553 & 0.0584 & 1.3444 & 0.0687 & 0.0671 & 0.9898 & 0.0688 & 0.0630 \\
\hline & & $\theta$ & 1.4032 & 0.0698 & 0.0697 & 1.3988 & 0.0704 & 0.0692 & 1.3573 & 0.0674 & 0.0642 \\
\hline & \multirow{3}{*}{$(50,45)$} & $\alpha$ & 1.4527 & 0.0721 & 0.0744 & 1.3228 & 0.0666 & 0.0653 & 1.1848 & 0.0651 & 0.0644 \\
\hline & & $\gamma$ & 1.6195 & 0.0799 & 0.0985 & 0.9865 & 0.0493 & 0.0489 & 1.0898 & 0.0505 & 0.0507 \\
\hline & & $\theta$ & 1.2685 & 0.0635 & 0.0642 & 1.1417 & 0.0536 & 0.0549 & 1.1424 & 0.0568 & 0.0565 \\
\hline \multirow{6}{*}{0.75} & \multirow{3}{*}{$(50,30)$} & $\alpha$ & 1.5826 & 0.0603 & 0.0624 & 1.6656 & 0.0586 & 0.0633 & 1.3443 & 0.0619 & 0.0625 \\
\hline & & $\gamma$ & 1.1616 & 0.0444 & 0.0447 & 1.4141 & 0.0520 & 0.0541 & 1.0675 & 0.0517 & 0.0548 \\
\hline & & $\theta$ & 1.4538 & 0.0540 & 0.0552 & 1.4689 & 0.0561 & 0.0571 & 1.4140 & 0.0540 & 0.0565 \\
\hline & \multirow{3}{*}{$(50,45)$} & $\alpha$ & 1.8404 & 0.0827 & 0.0873 & 1.4767 & 0.0653 & 0.0662 & 1.2576 & 0.0677 & 0.0668 \\
\hline & & $\gamma$ & 1.9936 & 0.0852 & 0.1015 & 1.1505 & 0.0467 & 0.0528 & 1.3852 & 0.0531 & 0.0524 \\
\hline & & $\theta$ & 1.1563 & 0.0517 & 0.0501 & 1.0515 & 0.0481 & 0.0482 & 1.0972 & 0.0475 & 0.0496 \\
\hline \multirow{6}{*}{0.25} & \multirow{3}{*}{$(150,90)$} & $\alpha$ & 3.4917 & 0.1996 & 0.1972 & 2.0333 & 0.1130 & 0.1154 & 1.4602 & 0.1115 & 0.1126 \\
\hline & & $\gamma$ & 3.0419 & 0.1640 & 0.1818 & 1.2409 & 0.0715 & 0.0645 & 1.7235 & 0.0683 & 0.0679 \\
\hline & & $\theta$ & 1.1229 & 0.0584 & 0.0649 & 0.8407 & 0.0458 & 0.0443 & 0.9857 & 0.0452 & 0.0465 \\
\hline & \multirow{3}{*}{$(150,130)$} & $\alpha$ & 3.4100 & 0.2276 & 0.3452 & 1.1130 & 0.0823 & 0.0866 & 1.1313 & 0.0849 & 0.0826 \\
\hline & & $\gamma$ & 2.9281 & 0.2013 & 0.3400 & 0.7831 & 0.0562 & 0.0590 & 1.4864 & 0.0579 & 0.0569 \\
\hline & & $\theta$ & 1.0169 & 0.0798 & 0.0865 & 0.6260 & 0.0465 & 0.0477 & 0.8743 & 0.0469 & 0.0494 \\
\hline \multirow{6}{*}{0.5} & \multirow{3}{*}{$(150,90)$} & $\alpha$ & 3.0771 & 0.2313 & 0.2694 & 1.6110 & 0.1245 & 0.1253 & 1.3156 & 0.1271 & 0.1248 \\
\hline & & $\gamma$ & 2.1543 & 0.1644 & 0.2014 & 1.1711 & 0.0838 & 0.0867 & 1.1085 & 0.0873 & 0.0934 \\
\hline & & $\theta$ & 1.2831 & 0.0975 & 0.1007 & 1.0304 & 0.0785 & 0.0838 & 1.0378 & 0.0803 & 0.0789 \\
\hline & \multirow{3}{*}{$(150,130)$} & $\alpha$ & 2.8276 & 0.2045 & 0.2572 & 1.2477 & 0.0784 & 0.0727 & 1.8901 & 0.0782 & 0.0708 \\
\hline & & $\gamma$ & 3.0523 & 0.1741 & 0.2241 & 0.9132 & 0.0570 & 0.0548 & 1.1321 & 0.0540 & 0.0575 \\
\hline & & $\theta$ & 1.0939 & 0.0669 & 0.0682 & 0.6853 & 0.0407 & 0.0408 & 0.7206 & 0.0401 & 0.0413 \\
\hline \multirow{6}{*}{0.75} & \multirow{3}{*}{$(150,90)$} & $\alpha$ & 3.3820 & 0.2318 & 0.2783 & 1.6746 & 0.1197 & 0.1175 & 1.2440 & 0.1136 & 0.1178 \\
\hline & & $\gamma$ & 3.9865 & 0.2525 & 0.3649 & 1.4019 & 0.0999 & 0.0967 & 1.6823 & 0.0977 & 0.1047 \\
\hline & & $\theta$ & 1.2103 & 0.0783 & 0.0873 & 1.0119 & 0.0738 & 0.0681 & 1.0804 & 0.0707 & 0.0717 \\
\hline & \multirow{3}{*}{$(150,130)$} & $\alpha$ & 0.8889 & 0.0782 & 0.0832 & 1.1486 & 0.1104 & 0.1037 & 0.8854 & 0.1011 & 0.0986 \\
\hline & & $\gamma$ & 0.7358 & 0.0675 & 0.0690 & 0.8441 & 0.0698 & 0.0746 & 0.8303 & 0.0758 & 0.0712 \\
\hline & & $\theta$ & 0.7044 & 0.0631 & 0.0642 & 0.6814 & 0.0603 & 0.0616 & 0.7032 & 0.0634 & 0.0588 \\
\hline
\end{tabular}

(1) Start with any initial values $\left(\Theta_{l}^{0}\right) ; \Theta=(\alpha, \gamma, \theta) ; l=1,2,3$ satisfying $\pi\left(\Theta_{l}^{0}\right)>0$.

(2) Using the initial value, sample a candidate point $\left(\Theta^{*}\right)$ from the proposal $q\left(\Theta^{*}\right)$.

(3) For $t=0$ to $\mathrm{N}$ (a huge number 10,000, for example), given the candidate point $\left(\Theta^{*}\right)$, calculate the acceptance probability $A_{l}=\min \left(1,\left(L_{1}\left(\Theta_{l}^{*} \mid x\right) \pi\left(\Theta_{l}^{*}\right) / L_{1}\left(x \mid \Theta_{l}\right) \pi\left(\Theta_{l}\right)\right) q\left(\Theta_{l}\right) / q\left(\Theta_{l}^{*}\right)\right) ; l=1,2,3$.

(4) Draw a value $u$ from the uniform $(0,1)$ distribution $\Theta_{l}^{t+1}=\left\{\begin{array}{l}\Theta_{l}^{*} \text { if } u \leq A_{l} \\ \Theta_{l}^{t} \text { if } u \leq A_{l}\end{array}\right.$.

(5) Repeat steps $2-4, t+1$ times until we get $N$ draws.

(6) The Bayes estimate of $\Theta_{l}$, with respect to squared error loss function is $\sum_{t=1}^{N}\left(\left(\Theta_{l}^{t-1}\right)_{t} / N\right)$.

(7) Repeat the above steps $l$ times to get a Bayesian estimate of $\Theta_{l}$.

Algorithm 1

(1) Arrange $\Theta_{l}{ }^{j} ; l=1,2,3$ as $\alpha^{[1]}, \alpha^{[2]}, \ldots, \alpha^{[L]}, \gamma^{[1]}, \gamma^{[2]}, \ldots, \gamma^{[L]}$ and $\theta^{[1]}, \theta^{[2]}, \ldots, \theta^{[L]}$ where $L$ is the length of simulation generated.

(2) The $95 \%$ symmetric credible intervals of $\alpha, \gamma$, and $\theta$ become $\left(\alpha^{[L(0.05 / 2)]}, \alpha^{[L(1-(0.05 / 2))]}\right),\left(\gamma^{[L(0.05 / 2)]}, \gamma^{[L(1-(0.05 / 2))]}\right)$ and $\left(\theta^{[L(0.05 / 2)]}, \theta^{[L(1-(0.05 / 2))]}\right)$. 
TABLE 3: Parameter estimation for WGED under ATIIPCS with random removals in case-1.

\begin{tabular}{|c|c|c|c|c|c|c|c|c|c|c|c|}
\hline \multicolumn{12}{|c|}{$\alpha=3.5, \gamma=3, \theta=2.5$, and $T=0.25$} \\
\hline \multirow{2}{*}{$P$} & \multirow{2}{*}{$(n, m)$} & & \multicolumn{2}{|c|}{ MLE } & \multicolumn{2}{|c|}{ MPS } & \multicolumn{2}{|c|}{ Bayesian } & \multicolumn{3}{|c|}{ Relative efficiency } \\
\hline & & & Bias & MSE & Bias & MSE & Bias & MSE & $\mathrm{RE}_{1}$ & $\mathrm{RE}_{2}$ & $\mathrm{RE}_{3}$ \\
\hline \multirow{6}{*}{0.25} & \multirow{3}{*}{$(50,30)$} & $\alpha$ & -0.0353 & 1.6448 & -0.0584 & 0.5179 & 0.0273 & 0.3462 & 3.1949 & 4.7573 & 1.4891 \\
\hline & & $\gamma$ & 0.0751 & 0.2288 & -0.0377 & 0.1305 & 0.0343 & 0.0575 & 1.7294 & 3.9595 & 2.2895 \\
\hline & & $\theta$ & 0.3323 & 0.2814 & 0.1060 & 0.1486 & 0.3238 & 0.2556 & 1.2454 & 1.1345 & 0.9110 \\
\hline & \multirow{3}{*}{$(50,45)$} & $\alpha$ & -0.0759 & 0.3302 & -0.1571 & 0.5090 & -0.0402 & 0.2033 & 0.6699 & 1.6083 & 2.4008 \\
\hline & & $\gamma$ & 0.0848 & 0.0878 & 0.0299 & 0.1154 & 0.0517 & 0.0434 & 0.7042 & 1.9815 & 2.8141 \\
\hline & & $\theta$ & 0.1029 & 0.1017 & -0.0737 & 0.0863 & 0.0982 & 0.0933 & 1.1270 & 1.0886 & 0.9659 \\
\hline \multirow{6}{*}{0.5} & \multirow{3}{*}{$(50,30)$} & $\alpha$ & 0.0329 & 0.4587 & -0.0098 & 0.5294 & 0.0411 & 0.2835 & 0.8645 & 1.6236 & 1.8781 \\
\hline & & $\gamma$ & 0.0758 & 0.0974 & -0.0241 & 0.1210 & 0.0490 & 0.0562 & 0.7612 & 1.7053 & 2.2401 \\
\hline & & $\theta$ & 0.3011 & 0.2671 & 0.0759 & 0.1560 & 0.2917 & 0.2473 & 1.1744 & 1.0879 & 0.9264 \\
\hline & \multirow{3}{*}{$(50,45)$} & $\alpha$ & -0.1250 & 1.9345 & -0.1581 & 0.5800 & -0.0344 & 0.2178 & 3.4576 & 8.8582 & 2.5619 \\
\hline & & $\gamma$ & 0.1038 & 0.2630 & 0.0405 & 0.1420 & 0.0507 & 0.0404 & 1.7964 & 6.6583 & 3.7065 \\
\hline & & $\theta$ & 0.0910 & 0.1060 & -0.0822 & 0.0885 & 0.0883 & 0.0920 & 1.1947 & 1.1598 & 0.9707 \\
\hline \multirow{6}{*}{0.75} & \multirow{3}{*}{$(50,30)$} & $\alpha$ & 0.0158 & 0.9863 & 0.0080 & 0.4823 & 0.0518 & 0.3242 & 2.0446 & 3.0663 & 1.4997 \\
\hline & & $\gamma$ & 0.0922 & 0.1620 & -0.0262 & 0.1059 & 0.0574 & 0.0662 & 1.4589 & 2.4400 & 1.6725 \\
\hline & & $\theta$ & 0.2629 & 0.2467 & 0.0473 & 0.1480 & 0.2582 & 0.2245 & 1.2181 & 1.1249 & 0.9235 \\
\hline & \multirow{3}{*}{$(50,45)$} & $\alpha$ & -0.0934 & 0.3563 & -0.1708 & 0.4958 & -0.0130 & 0.1929 & 0.7448 & 1.8034 & 2.4211 \\
\hline & & $\gamma$ & 0.0807 & 0.0824 & 0.0237 & 0.1047 & 0.0375 & 0.0371 & 0.7290 & 2.1300 & 2.9218 \\
\hline & & $\theta$ & 0.0907 & 0.0989 & -0.0842 & 0.0871 & 0.0857 & 0.0910 & 1.1321 & 1.0827 & 0.9563 \\
\hline \multirow{6}{*}{0.25} & \multirow{3}{*}{$(150,90)$} & $\alpha$ & 0.2779 & 2.8751 & 0.3296 & 1.1280 & 0.1102 & 0.4102 & 2.7446 & 7.0288 & 2.5609 \\
\hline & & $\gamma$ & 0.0130 & 0.2429 & -0.0578 & 0.0775 & 0.0195 & 0.0301 & 3.2746 & 8.1563 & 2.4908 \\
\hline & & $\theta$ & 0.1559 & 0.0755 & 0.0635 & 0.0452 & 0.1544 & 0.0663 & 1.2443 & 1.2063 & 0.9694 \\
\hline & \multirow{3}{*}{$(150,130)$} & $\alpha$ & 0.0168 & 0.0697 & -0.0132 & 0.1133 & -0.0056 & 0.1705 & 0.6134 & 0.4071 & 0.6636 \\
\hline & & $\gamma$ & 0.0183 & 0.0158 & -0.0098 & 0.0281 & 0.0240 & 0.0196 & 0.5532 & 0.8122 & 1.4682 \\
\hline & & $\theta$ & 0.0593 & 0.0332 & -0.0188 & 0.0289 & 0.0569 & 0.0315 & 1.0384 & 1.0519 & 1.0131 \\
\hline \multirow{6}{*}{0.5} & \multirow{3}{*}{$(150,90)$} & $\alpha$ & 0.3311 & 1.0983 & 0.3788 & 1.2220 & 0.1473 & 0.3313 & 0.9168 & 3.1933 & 3.4830 \\
\hline & & $\gamma$ & -0.0078 & 0.0783 & -0.0689 & 0.0903 & 0.0043 & 0.0307 & 0.9141 & 2.5497 & 2.7894 \\
\hline & & $\theta$ & 0.1254 & 0.0625 & 0.0310 & 0.0445 & 0.1218 & 0.0600 & 1.0736 & 1.0359 & 0.9648 \\
\hline & \multirow{3}{*}{$(150,130)$} & $\alpha$ & -0.0780 & 0.3891 & -0.0168 & 0.1021 & -0.0325 & 0.1964 & 3.7041 & 2.6819 & 0.5212 \\
\hline & & $\gamma$ & 0.0415 & 0.0440 & -0.0120 & 0.0281 & 0.0306 & 0.0218 & 1.9674 & 2.9887 & 1.3391 \\
\hline & & $\theta$ & 0.0458 & 0.0384 & -0.0293 & 0.0293 & 0.0455 & 0.0306 & 1.2752 & 1.2713 & 0.9969 \\
\hline \multirow{6}{*}{0.75} & \multirow{3}{*}{$(150,90)$} & $\alpha$ & 0.3233 & 0.5615 & 0.3882 & 0.1556 & 0.1340 & 0.3630 & 1.4498 & 4.2225 & 2.9124 \\
\hline & & $\gamma$ & 0.0023 & 0.2837 & -0.0770 & 0.0727 & 0.0133 & 0.0608 & 3.2485 & 2.6787 & 1.1013 \\
\hline & & $\theta$ & 0.1190 & 0.0674 & 0.0281 & 0.0447 & 0.1154 & 0.0614 & 1.2112 & 1.1073 & 0.9142 \\
\hline & & $\alpha$ & -0.0111 & 0.1078 & -0.0455 & 0.1691 & -0.0123 & 0.1776 & 0.6446 & 0.6067 & 0.9412 \\
\hline & $(150,130)$ & $\gamma$ & 0.0262 & 0.0283 & 0.0018 & 0.0467 & 0.0229 & 0.0215 & 0.5920 & 1.3195 & 2.2289 \\
\hline & & $\theta$ & 0.0333 & 0.0309 & -0.0447 & 0.0309 & 0.0317 & 0.0293 & 1.0290 & 1.0511 & 1.0215 \\
\hline
\end{tabular}

$2,3,1,1,2,0^{(10)}$. The $x_{i: m: n}$ is $0.101,0.332,0.403,0.550,0.596$, $0.597,0.645,0.654,0.722,0.859,1.056,1.117,1.128,1.196$, $1.325,1.532,1.577,1.701,1.754$, and 2.052 . The time of the adaptive model is 1.5. By using the KS test and MLE estimate, the distance of the KS test is 0.1451 , and the $p$ value is 0.133 . If $p=0.5, m=20$, and the time of the adaptive model is 1.5 , then the distance of the KS test is 0.13734 , and the $p$ value is 0.1756 . If $p=0.25, m=50$, and the time of the adaptive model is 1.5 , then the distance of the KS test is 0.07134 , and the $p$ value is 0.8882 . And if $p=0.5, m=50$, and the time of the adaptive model is 1.5 , then the distance of the KS test is 0.0784 , and the $p$ value is 0.8121 . Therefore, it indicates that WGED can be fitted to the data set based on APTIIC. Table 9 shows estimation of parameters and standard error for WGED based on ATIIPCS for carbon fiber data when $m=20$, $p=0.25$, and $T=1.5$. 
TABLE 4: Parameter estimation for WGED under ATIIPCS with random removals in case-2.

\begin{tabular}{|c|c|c|c|c|c|c|c|c|c|c|c|}
\hline & & & & & $0.5, \gamma=2$ & $=2.5$, an & $=0.5$ & & & & \\
\hline$P$ & & & & & & & Bay & & & ive effici & \\
\hline$P$ & $(n, m)$ & & Bias & MSE & Bias & MSE & Bias & MSE & RE1 & RE2 & RE3 \\
\hline & & $\alpha$ & -0.0321 & 0.5181 & 0.0489 & 0.1566 & 0.1109 & 0.1648 & 3.3530 & 3.3901 & 1.0111 \\
\hline & $(50,30)$ & $\gamma$ & 0.0445 & 0.4443 & -0.0061 & 0.1126 & -0.0237 & 0.1597 & 3.9283 & 2.7804 & 0.7078 \\
\hline & & $\theta$ & 0.3359 & 0.2987 & 0.1195 & 0.1690 & 0.3290 & 0.2723 & 1.2009 & 1.1329 & 0.9434 \\
\hline 0.25 & & $\alpha$ & 0.0775 & 0.1357 & 0.1566 & 0.2282 & 0.1771 & 0.1406 & 0.6366 & 1.1870 & 1.8646 \\
\hline & $(50,45)$ & $\gamma$ & -0.0169 & 0.0599 & -0.0493 & 0.0778 & -0.0669 & 0.0598 & 0.7904 & 1.0773 & 1.3630 \\
\hline & & $\theta$ & 0.1073 & 0.0816 & -0.0630 & 0.0690 & 0.1064 & 0.0774 & 1.0774 & 1.0603 & 0.9842 \\
\hline & & $\alpha$ & 0.0746 & 0.1928 & 0.1303 & 0.2260 & 0.1120 & 0.1196 & 0.8958 & 1.7499 & 1.9534 \\
\hline & $(50,30)$ & $\gamma$ & -0.0249 & 0.0886 & -0.0453 & 0.1194 & -0.0457 & 0.0657 & 0.7500 & 1.3836 & 1.8448 \\
\hline & & $\theta$ & 0.2984 & 0.2169 & 0.0721 & 0.1322 & 0.2896 & 0.2035 & 1.0064 & 1.0688 & 1.0620 \\
\hline 0.5 & & $\alpha$ & 0.0411 & 0.1387 & 0.0891 & 0.1215 & 0.1289 & 0.1077 & 1.2062 & 1.5034 & 1.2464 \\
\hline & $(50,45)$ & $\gamma$ & 0.0230 & 0.0708 & -0.0097 & 0.0633 & -0.0145 & 0.0773 & 1.6951 & 1.2084 & 0.8194 \\
\hline & & $\theta$ & 0.1069 & 0.1159 & -0.0630 & 0.0886 & 0.1093 & 0.0967 & 1.2344 & 1.2329 & 0.9988 \\
\hline & & $\alpha$ & 0.0545 & 0.1657 & 0.1114 & 0.1927 & 0.1275 & 0.1337 & 0.9028 & 1.3861 & 1.5353 \\
\hline & $(50,30)$ & $\gamma$ & 0.0078 & 0.0877 & -0.0108 & 0.1301 & -0.0277 & 0.0748 & 0.6747 & 1.1841 & 1.7550 \\
\hline & & $\theta$ & 0.2755 & 0.2132 & 0.0527 & 0.1430 & 0.2675 & 0.2015 & 0.9795 & 1.0570 & 1.0792 \\
\hline 0.75 & & $\alpha$ & 0.0403 & 0.1216 & 0.1024 & 0.1521 & 0.1191 & 0.1169 & 1.5531 & 2.1416 & 1.3789 \\
\hline & $(50,45)$ & $\gamma$ & 0.0345 & 0.0593 & 0.0010 & 0.0860 & 0.0137 & 0.0248 & 3.0025 & 2.0714 & 1.6899 \\
\hline & & $\theta$ & 0.0704 & 0.0918 & -0.0986 & 0.0815 & 0.0704 & 0.0832 & 1.2093 & 1.1106 & 0.9184 \\
\hline & & $\alpha$ & 0.3257 & 0.8972 & 0.3340 & 0.3798 & 0.2828 & 0.2183 & 2.9491 & 5.7178 & 1.9388 \\
\hline & $(150,90)$ & $\gamma$ & -0.1838 & 0.6342 & -0.2081 & 0.1432 & -0.1475 & 0.2145 & 6.0092 & 3.1150 & 0.5184 \\
\hline & & $\theta$ & 0.1167 & 0.0954 & 0.0259 & 0.0465 & 0.1145 & 0.0762 & 1.7842 & 1.2978 & 0.7274 \\
\hline 0.25 & & $\alpha$ & -0.0282 & 0.7532 & 0.0615 & 0.0839 & 0.1177 & 0.0967 & 9.3874 & 9.0850 & 0.9678 \\
\hline & $(150,130)$ & $\gamma$ & 0.0447 & 0.5568 & -0.0177 & 0.0400 & 0.0072 & 0.1430 & 13.9802 & 3.8808 & 0.2776 \\
\hline & & $\theta$ & 0.0644 & 0.0711 & -0.0002 & 0.0254 & 0.0670 & 0.0540 & 2.6390 & 1.3528 & 0.5126 \\
\hline & & $\alpha$ & 0.1114 & 0.6250 & 0.1539 & 0.1916 & 0.1422 & 0.1322 & 3.6481 & 5.4704 & 1.4995 \\
\hline & $(150,90)$ & $\gamma$ & -0.0210 & 0.3007 & -0.0603 & 0.0924 & -0.0329 & 0.0806 & 3.3839 & 3.7766 & 1.1161 \\
\hline & & $\theta$ & 0.1470 & 0.1281 & 0.0595 & 0.0722 & 0.1518 & 0.0927 & 1.5507 & 1.5286 & 0.9857 \\
\hline 0.5 & & $\alpha$ & -0.0632 & 0.4375 & 0.0902 & 0.1091 & 0.1130 & 0.1249 & 3.0109 & 4.8205 & 0.4358 \\
\hline & $(150,130)$ & $\gamma$ & 0.0460 & 0.6063 & -0.0195 & 0.0545 & -0.0021 & 0.0783 & 3.1722 & 3.2697 & 0.6507 \\
\hline & & $\theta$ & 0.0328 & 0.0787 & -0.0307 & 0.0314 & 0.0490 & 0.0361 & 2.5475 & 2.3045 & 0.9046 \\
\hline & & $\alpha$ & 0.1431 & 0.7612 & 0.1730 & 0.2115 & 0.1684 & 0.1286 & 4.0788 & 7.3907 & 1.8120 \\
\hline & $(150,90)$ & $\gamma$ & -0.0199 & 1.0295 & -0.0541 & 0.1302 & -0.0391 & 0.1848 & 8.0864 & 5.6152 & 0.6944 \\
\hline 07 & & $\theta$ & 0.1651 & 0.1221 & 0.0592 & 0.0698 & 0.1622 & 0.1019 & 1.4304 & 1.2549 & 0.8773 \\
\hline 0.75 & & $\alpha$ & 0.0372 & 0.0524 & 0.0666 & 0.0896 & 0.0802 & 0.0570 & 0.5989 & 1.0081 & 1.6830 \\
\hline & $(150,130)$ & $\gamma$ & -0.0063 & 0.0350 & -0.0109 & 0.0461 & 0.0045 & 0.0445 & 0.7597 & 0.7853 & 1.0337 \\
\hline & & $\theta$ & 0.0668 & 0.0365 & -0.0149 & 0.0302 & 0.0661 & 0.0363 & 1.0687 & 1.0035 & 0.9390 \\
\hline
\end{tabular}

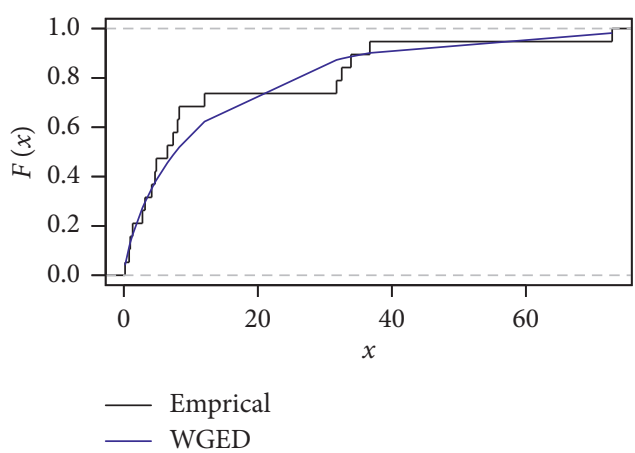

(a)

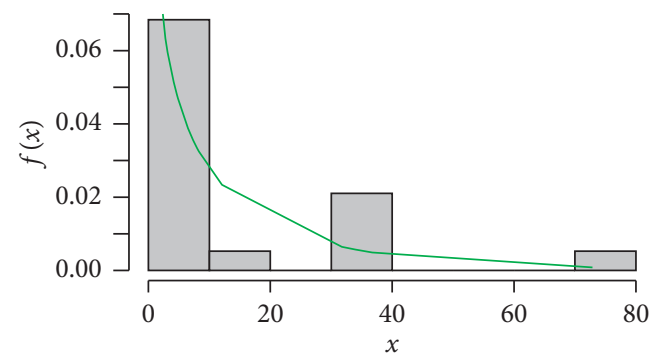

Figure 1: Continued. 


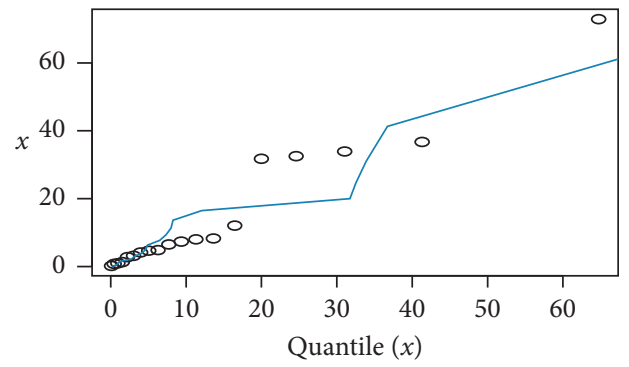

(c)

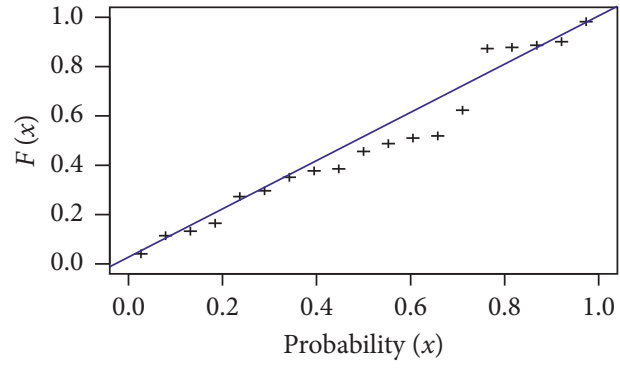

(d)

FIGURe 1: Plot of the max distance between two ECDF curves, histogram, PP-plot, and QQ-plot of WGED for electric data.

TABLE 5: Estimation of parameters and standard error for complete electric data.

\begin{tabular}{cccccrr}
\hline & \multicolumn{2}{c}{ MLE } & \multicolumn{2}{c}{ MPS } & \multicolumn{2}{c}{ Bayesian } \\
& Estimate & St.E & Estimate & St.E & Estimate \\
\hline$\alpha$ & 15.1210 & 0.0002 & 15.3220 & 0.0001 & 15.0352 & 0.0887 \\
$\gamma$ & 0.0022 & 0.0012 & 0.0013 & 0.0009 & 0.00208 & 0.0011 \\
$\theta$ & 0.7565 & 0.1263 & 0.6623 & 0.1190 & 0.6676 & 0.0842 \\
\hline
\end{tabular}

TABle 6: Data of progressive Type-II censored data with censoring for electric data.

\begin{tabular}{lcccccccc}
\hline$i$ & 1 & 2 & 3 & 4 & 5 & 6 & 7 & 8 \\
\hline$R$ & 0 & 0 & 3 & 0 & 3 & 0 & 0 & 5 \\
$x_{i: m: n}$ & 0.19 & 0.78 & 0.96 & 1.31 & 2.7 & 4.85 & 6.50 & 7.35 \\
\hline
\end{tabular}

TABLE 7: Estimation of parameters and standard error under the censored sample for electric data.

\begin{tabular}{ccccccr}
\hline & \multicolumn{2}{c}{ MLE } & & MPS & \multicolumn{2}{c}{ Bayesian } \\
& St.E & Estimate & St.E & Estimate & St.E & 0.4883 \\
$\gamma \alpha$ & 0.4656 & 1.2150 & 0.4009 & 0.8323 & 0.2438 \\
$\gamma$ & 0.1535 & 0.3445 & 0.1785 & 0.4064 & 0.1062 \\
$\theta$ & 0.7808 & 0.4457 & 0.5950 & 0.3781 & 0.5964 & 0.2064 \\
\hline
\end{tabular}
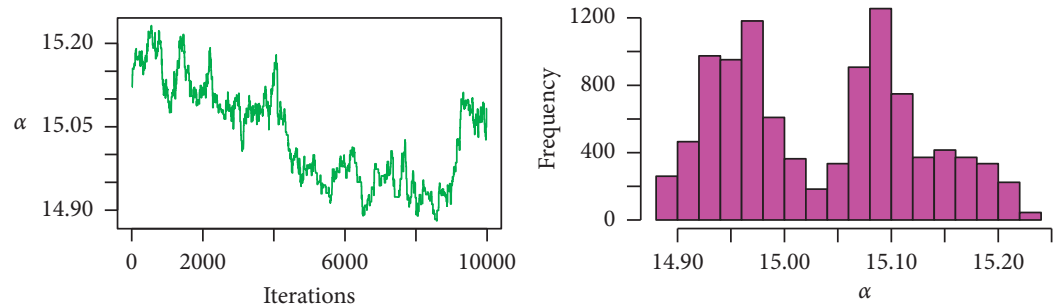

(a)
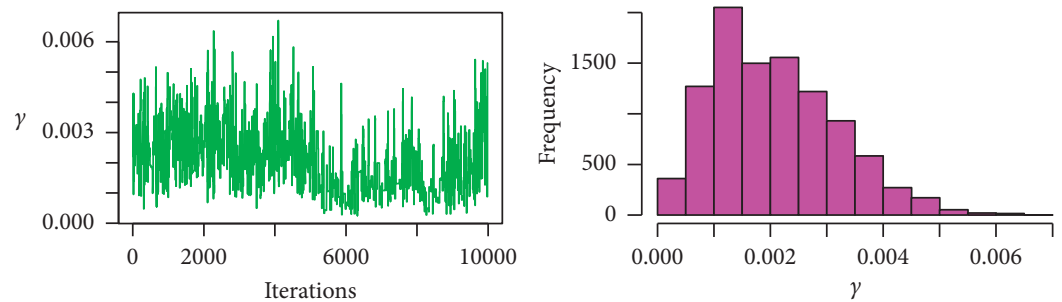

(b)
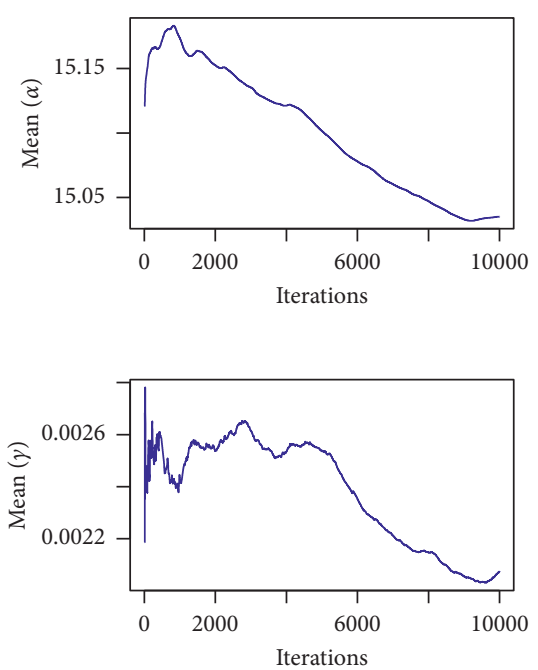

FIgURE 2: Continued. 

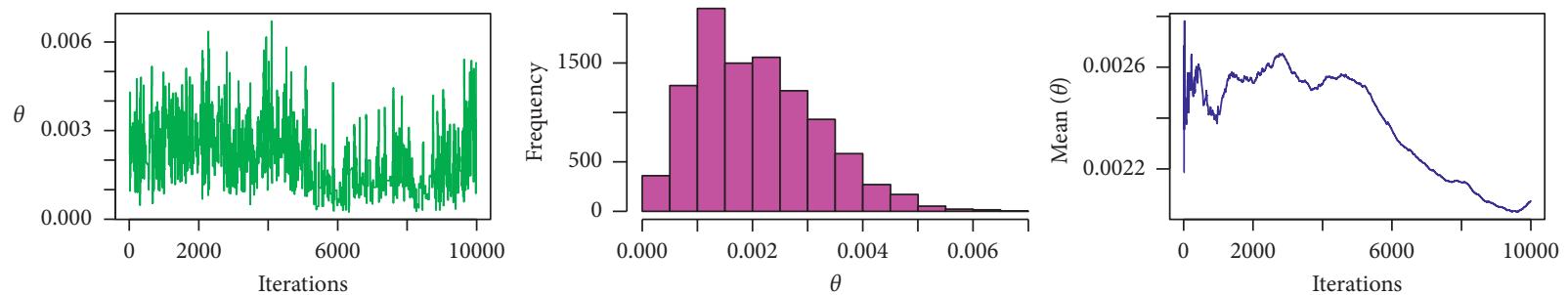

(c)

FIGURE 2: The MCMC plots are based on a complete sample of WGED for electric data.
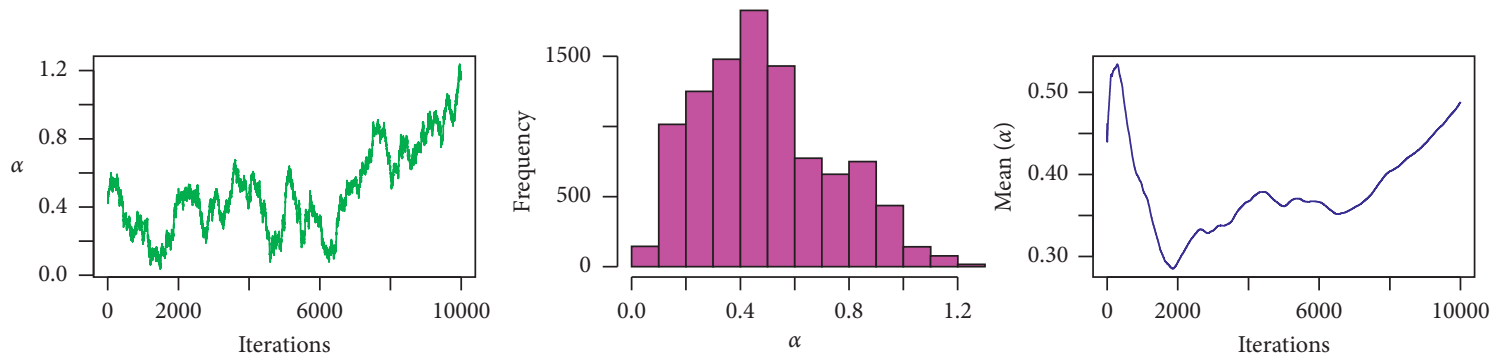

(a)
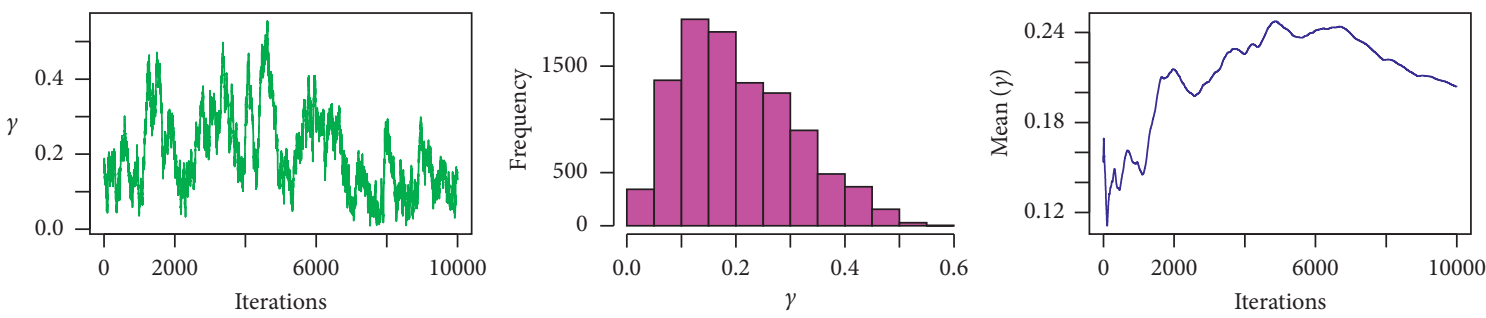

(b)
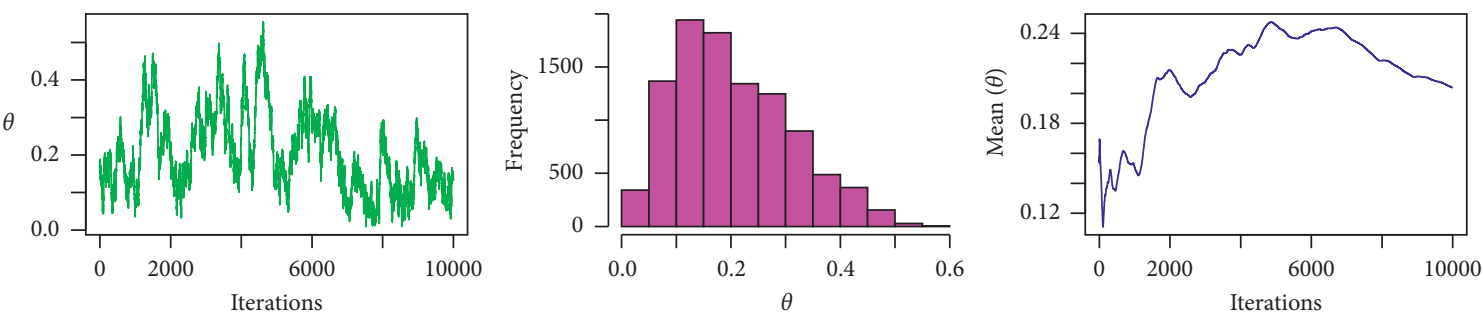

(c)

FIgURE 3: The MCMC plots based on a censored sample of WGED for electric data.

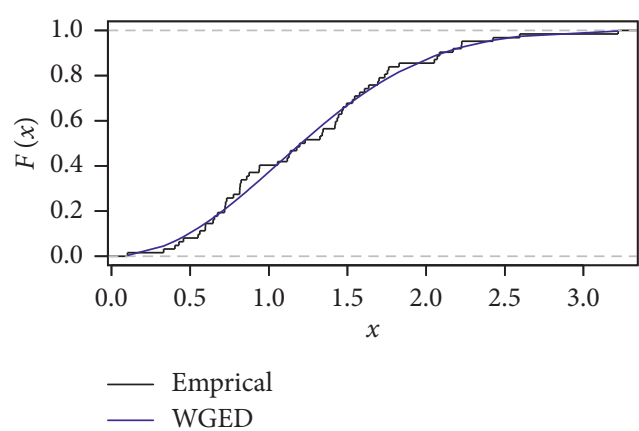

(a)

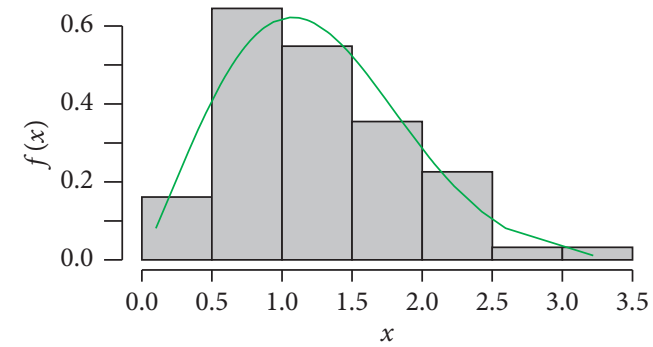

Figure 4: Continued.

(b) 


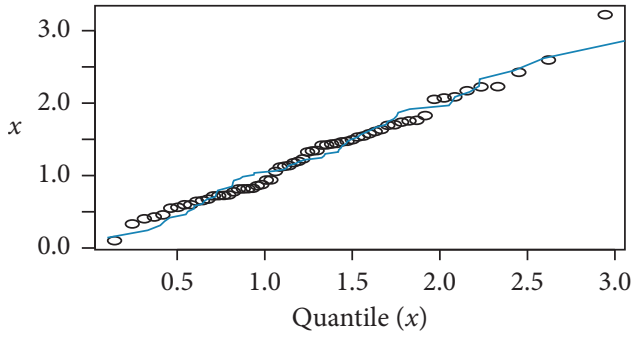

(c)

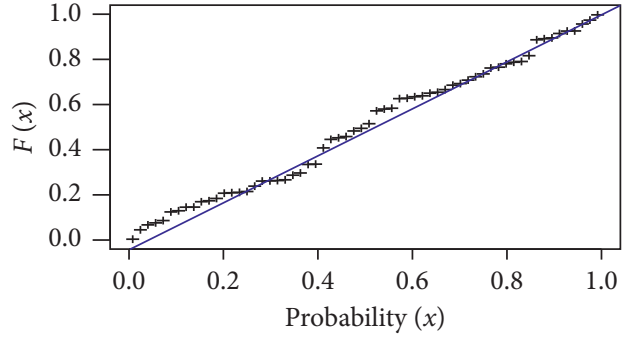

(d)

Figure 4: Plot of the max distance between two ECDF curves, histogram, PP-plot, and QQ-plot for carbon fiber data.

TABLE 8: Estimation of parameters and standard error for complete carbon fiber data.

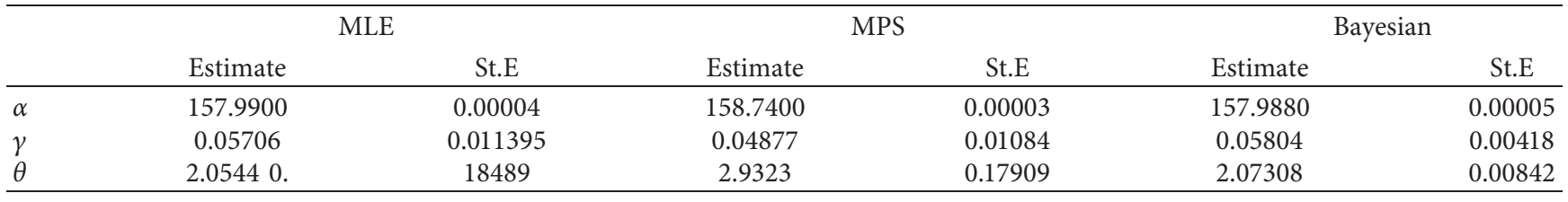

TABLE 9: Estimation and standard error for WGED based on ATIIPCS for carbon fiber data when $m=20,50, p=0.25,0.5$, and $T=1.5$.

\begin{tabular}{|c|c|c|c|c|c|c|c|c|}
\hline \multirow{2}{*}{$m$} & \multirow{2}{*}{$P$} & & \multicolumn{2}{|c|}{ MLE } & \multicolumn{2}{|c|}{ MPS } & \multicolumn{2}{|c|}{ Bayesian } \\
\hline & & & Estimate & St.E & Estimate & St.E & Estimate & St.E \\
\hline \multirow{6}{*}{20} & \multirow{3}{*}{0.25} & $\alpha$ & 0.9001 & 4.0209 & 0.61040 & 2.2957 & 0.7170 & 0.6955 \\
\hline & & $\gamma$ & 0.5491 & 0.9001 & 0.62583 & 0.9917 & 0.6955 & 0.2640 \\
\hline & & $\theta$ & 1.9092 & 0.8464 & 1.64328 & 0.7814 & 1.7813 & 0.3383 \\
\hline & \multirow{3}{*}{0.5} & $\alpha$ & 36.7260 & 0.0003 & 36.729 & 0.0002 & 36.7269 & 0.0096 \\
\hline & & $\gamma$ & 0.0851 & 0.0204 & 0.06707 & 0.0197 & 0.0763 & 0.0200 \\
\hline & & $\theta$ & 1.8743 & 0.237 & 1.6899 & 0.2241 & 1.7765 & 0.2188 \\
\hline \multirow{6}{*}{50} & \multirow{3}{*}{0.25} & $\alpha$ & 65.2522 & 0.0001 & 65.2566 & 0.0002 & 65.2418 & 0.0001 \\
\hline & & $\gamma$ & 0.0828 & 0.0145 & 0.07128 & 0.0142 & 0.0637 & 0.1103 \\
\hline & & $\theta$ & 2.0128 & 0.1911 & 1.8779 & 0.1842 & 1.76834 & 0.1813 \\
\hline & \multirow{3}{*}{0.5} & $\alpha$ & 80.5530 & 0.0001 & 80.5570 & 0.0001 & 80.5505 & 0.00007 \\
\hline & & $\gamma$ & 0.0767 & 0.0142 & 0.0657 & 0.0138 & 0.0457 & 0.0084 \\
\hline & & $\theta$ & 2.0160 & 0.0192 & 1.8826 & 0.1854 & 1.6629 & 0.0960 \\
\hline
\end{tabular}
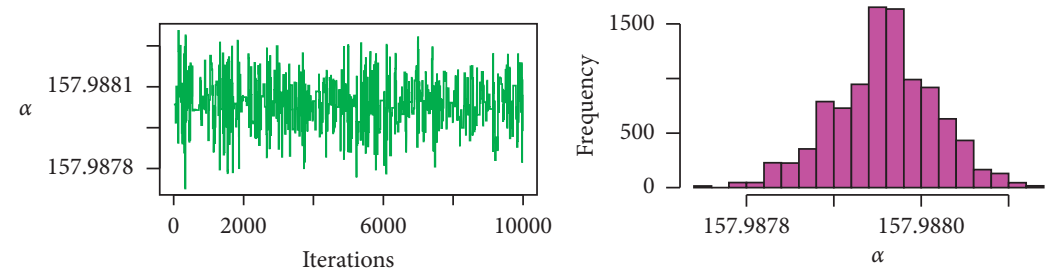

(a)
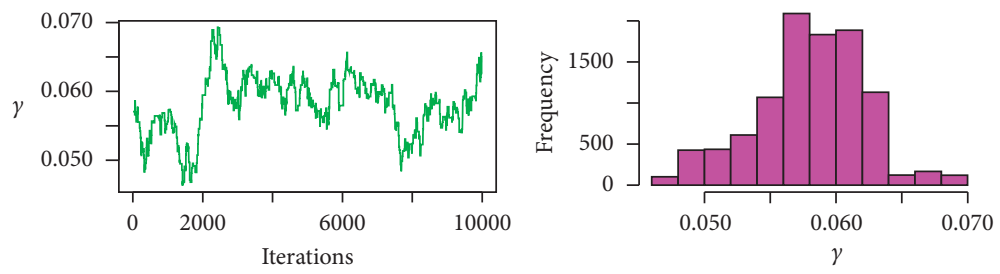

(b)
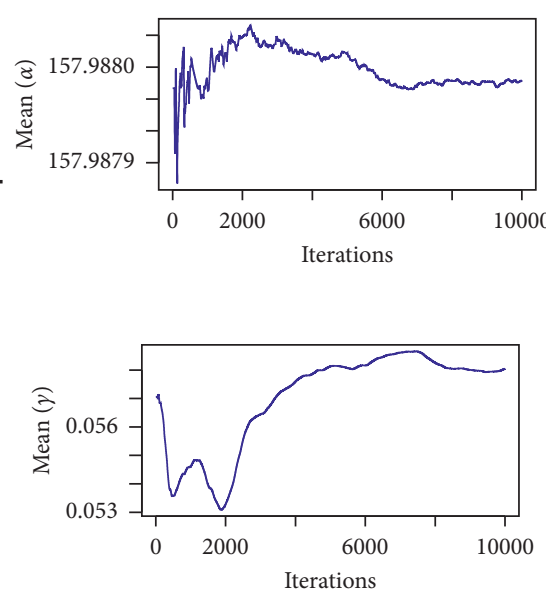

FIgURE 5: Continued. 

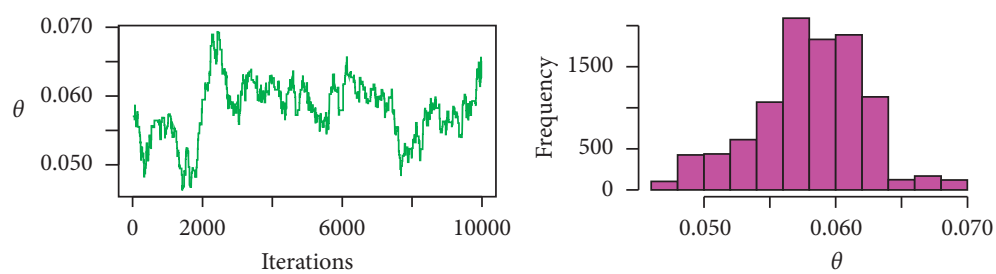

(c)

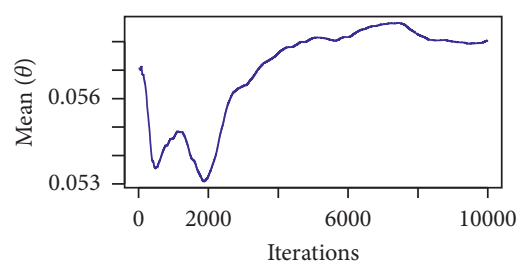

FIgURE 5: The MCMC plots are based on a complete sample of WGED for carbon fiber data.

\section{Conclusions}

In this paper, we discussed MLE, MPS, and Bayesian estimation to estimate the parameter problem of the WGED based on ATIIPCS with random removal. We used Bayesian estimation under the square error loss function to calculate the unknown parameters for WGED under the assumption of independent gamma priors. The performance of the different estimator optimal censoring schemes is compared based on a simulation study to determine the optimal censoring schemes by using MSE, the Bias, and the L.CI. It is noticeable that the Bayesian estimation is better and more efficient than the MLE and MPS estimation according to the MSE. We applied two real data applications based on ATIIPCS of carbon fiber and electric data which are obtained, we deduce that these sets provide an excellent fit for the proposed distribution according to the $p$ value, and also we plotted the PP-plots and other kinds of plots to make sure that the distribution is a good candidate to these real data sets.

\section{Data Availability}

The data used to support the findings of this study are included within the paper.

\section{Conflicts of Interest}

The authors declare that there are no conflicts of interest regarding the publication of this paper.

\section{Acknowledgments}

The authors are grateful to their universities.

\section{References}

[1] M. Bourguignon, R. B. Silva, and G. M. Cordeiro, "The Weibull-G family of probability distributions," Journal of Data Science, vol. 12no, pp. 53-68, 2014.

[2] A. Mustafa, B. S. El-Desouky, and S. AL-Garash, "Weibull generalized exponential distribution," 2016, http://arxiv.org/ abs/1606.07378.

[3] R. D. Gupta and D. Kundu, "Generalized exponential distributions," Australian, vol. 41, no. 2, pp. 173-188, 1999.

[4] S. Nadarajah and S. Kotz, "The beta exponential distribution," Reliability Engineering \& System Safety, vol. 91, no. 6, pp. 689-697, 2006.

[5] W. Barreto-Souza, A. H. Santos, and G. M. Cordeiro, "The beta generalized exponential distribution," Journal of
Statistical Computation and Simulation, vol. 80, no. 2, pp. 159-172, 2010.

[6] E. M. Almetwally, H. M. Almongy, and A. E. s. Mubarak, "Bayesian and maximum likelihood estimation for the Weibull generalized exponential distribution parameters using progressive censoring schemes," Pakistan Journal of Statistics and Operation Research, vol. 14, no. 4, pp. 853-868, 2018.

[7] N. Gupta and Q. A. Jamal, "Inference for Weibull generalized exponential distribution based on generalized order statistics," Journal of Applied Mathematics and Computing, vol. 61, no. $1-2$, pp. 573-592, 2019p.

[8] A. Z. Afify, A. M. Gemeay, and N. A. Ibrahim, "The heavytailed exponential distribution: risk measures, estimation, and application to actuarial data," Mathematics, vol. 8, no. 8, p. 1276, 2020.

[9] A.-E. A. M. Teamah, A. A. Elbanna, and A. M. Gemeay, "Frechet-Weibull mixture distribution: properties and applications," Applied Mathematical Sciences, vol. 14, no. 2, pp. 75-86, 2020.

[10] N. Balakrishnan and R. Aggarwala, Progressive Censoring: Theory, Methods, and Applications, Springer Science \& Business Media, Berlin, Germany, 2000.

[11] H. K. T. Ng, L. Luo, Y. Hu, and F. Duan, "Parameter estimation of three-parameter Weibull distribution based on progressively Type-II censored samples," Journal of Statistical Computation and Simulation, vol. 82, no. 11, pp. 1661-1678, 2012.

[12] S. Dey and T. Dey, "Statistical inference for the Rayleigh distribution under progressively Type-II censoring with binomial removal," Applied Mathematical Modelling, vol. 38, no. 3, pp. 974-982, 2014.

[13] E. M. Almetwally and H. M. Almongy, "Maximum product spacing and Bayesian method for parameter estimation for generalized power Weibull distribution under censoring scheme," Journal of Data Science, vol. 17, no. 2, pp. 407-444, 2019.

[14] M. Aslam, F. Noor, and S. Ali, "Shifted exponential distribution: bayesian estimation, prediction and expected test time under progressive censoring," Journal of Testing and Evaluation, vol. 48, no. 2, pp. 1576-1593, 2020.

[15] E.-S. A. El-Sherpieny, E. M. Almetwally, and H. Z. Muhammed, "Progressive Type-II hybrid censored schemes based on maximum product spacing with application to Power Lomax distribution," Physica A: Statistical Mechanics and Its Applications, vol. 553, p. 124251, 2020.

[16] H. K. T. Ng, D. Kundu, and P. S. Chan, "Statistical analysis of exponential lifetimes under an adaptive Type-II progressive censoring scheme," Naval Research Logistics (NRL), vol. 56, no. 8, pp. 687-698, $\square$ p2009.

[17] M. M. A. Sobhi and A. A. Soliman, "Estimation for the exponentiated Weibull model with adaptive Type-II progressive censored schemes," Applied Mathematical Modelling, vol. 40, no. 2, pp. 1180-2119, 2016. 
[18] S. F. Ateya and H. S. Mohammed, "Statistical inferences based on an adaptive progressive Type-II censoring from exponentiated exponential distribution," Journal of the Egyptian Mathematical Society, vol. 25, no. 4, pp. 393-399, 2017.

[19] E. M. Almetwally and H. M. Almongy, "Estimation of the marshall-olkin extended Weibull distribution parameters under adaptive censoring schemes," International Journal of Mathematical Archive, vol. 9, no. 9, pp. 95-102, 2018.

[20] E. M. Almetwally, H. M. Almongy, and M. H. Sabry, "Bayesian and classical estimation for the Weibull distribution parameters under progressive Type-II censoring schemes," International Journal of Mathematical Archive, vol. 10, no. 7, pp. 6-22, 2019.

[21] E. M. Almetwally, H. M. Almongy, M. K. Rastogi, and M. Ibrahim, "Maximum product spacing estimation of Weibull distribution under adaptive type-II progressive censoring schemes," Annals of Data Science, vol. 7, no. 2, pp. 257-279, 2020.

[22] Z. Yan and N. X. Wang, "Statistical analysis based on adaptive progressive hybrid censored sample from alpha power generalized exponential distribution," IEEE Access, vol. 8, pp. 54691-54697, 2020.

[23] S. Basu, S. K. Singh, and U. Singh, "Bayesian inference using product of spacings function for Progressive hybrid Type-I censoring scheme," Statistics, vol. 52, no. 2, pp. 345-363, 2018.

[24] R. Alshenawy, M. A. H. Sabry, E. M. Almetwally, and H. M. Elomngy, "Product spacing of stress-strength under progressive hybrid censored for exponentiated-gumbel distribution," Computers, Materials \& Continua, vol. 66, no. 3, pp. 2973-2995, 2021.

[25] R. Alshenawy, A. Al-Alwan, E. M. Almetwally, A. Z. Afify, and H. M. Almongy, "Progressive Type-II censoring schemes of extended odd Weibull exponential distribution with applications in medicine and engineering," Mathematics, vol. 8, no. 10, p. 1679, 2020.

[26] N. Metropolis, A. W. Rosenbluth, M. N. Rosenbluth, A. H. Teller, and E. Teller, "Equation of state calculations by fast computing machines," The Journal of Chemical Physics, vol. 21, no. 6, pp. 1087-1092, 1953.

[27] M. Z. Madi and M. T. Rabab, "Bayesian analysis for the exponentiated Rayleigh distribution," Metron - International Journal of Statistics, vol. 67, pp. 269-288, p2009.

[28] M. Nassar, O. Abo-Kasem, C. Zhang, and S. Dey, "Analysis of Weibull distribution under adaptive type-II progressive hybrid censoring scheme," Journal of the Indian Society for Probability and Statistics, vol. 19, no. 1, pp. 25-65, 2018.

[29] E. M. Almetwally, H. M. Almongy, and E. S. A. El-Sherpieny, "Adaptive type-II progressive censoring schemes based on maximum product spacing with application of generalized Rayleigh distribution," Journal of Data Science, vol. 17, no. 4, pp. 802-831, 2019.

[30] H. Haj Ahmad and E. Almetwally, "Marshall-olkin generalized pareto distribution: bayesian and non bayesian estimation," Pakistan Journal of Statistics and Operation Research, vol. 16, no. 1, pp. 21-33, 2020.

[31] B. Efron, "Bootstrap methods: another looks at the jackknife," in Breakthroughs in Statistics, pp. 569-593, Springer, New York, NY, USA, 1992.

[32] E. M. Almetwally, "Parameter estimation of bivariate models under some censoring schemes," Master thesis, Cairo University, Giza, Egypt, 2019.
[33] G. C. Canavos and C. P. Taokas, "Bayesian estimation of life parameters in the Weibull distribution," Operations Research, vol. 21, no. 3, pp. 755-763, 1973.

[34] M.-H. Chen and Q.-M. Shao, "Monte Carlo estimation of Bayesian credible and HPD intervals," Journal of Computational and Graphical Statistics, vol. 8, no. 1, pp. 69-92, 1999.

[35] N. Balakrishnan and E. Cramer, The Art of Progressive Censoring, Statistics for Industry and Technology, Giza, Egypt, 2014.

[36] M. G. Bader and A. M. Priest, "Statistical aspects of fibre and bundle strength in hybrid composites," Progress in Science and Engineering of Composites, pp. 1129-1136, 1982. 